Apidologie, 1975, 6 (1), 1-57.

\title{
LE PROBLĖMe DE L'ADAPTATION DE MEGACHILE (EUTRICHAREA) PACIFICA PANZ. (MEGACHILIDAE) AMÉRICAIN EN FRANCE
}

\author{
Zur Frage der Anpassung der aus Amerika stammenden Megachile \\ (Eutricharea) pacifica Panz. (Megachilidae) in Frankreich
}

\section{J.-N. TASEI}

avec la collaboration technique de F. de la condamine

Laboratoire d'Ethologie et d'Ecologie des Insectes, I.N.R.A., S.A.P.F. 86600 Lusignan

\author{
SUMMARY \\ ADAPTABILITY OF AMERICAN Megachile (Eutricharea) \\ pacifica PANZ. (Megachilidae) IN FRANCE
}

In 1972 and 1973 the author rears Megachile pacifica from Canada in lucerne fields of southeastern France for studying the multiplication rate of the American ecotype in French conditions. Removable grooved boards nesting traps are used according to the American method. Pinus and two African woods are tested : Entandrophragma and Terminalia. In most of the nesting sites the only first generation of females is allowed to found nests. Then the loss of cells in the progeny is estimated according to the site, the period of flight and the wood of trap. Total losses rates range between 50 and $95 \%$. The mortality due to natural indigenous enemies (mostly Trichodes apiarius) affects $10-50 \%$ of the cells. Pollen baits proove to be effective against Trichodes. Three to $35 \%$ of the eggs and larvae die for unknown reasons. The emergence rate of second generation adults reaches $11-64 \%$ of the cells. African woods are not favorable to larval development and increase the dormancy rate. Pinus shows opposite effects. If a second generation is allowed to nest, the third generation emergence rate is low $(6 \%)$. The weather conditions are not very favorable to female activity and the best population fold increase is 1,4 . In good shielding conditions one can expect to rear $M$. pacifica in the warmest districts of France.

\section{RÉSUME}

En 1972 et 1973 l'auteur élève dans les luzernières du Sud-Est de la France des Megachile pacifica importés du Canada, a fin de connaître les possibilités de multiplication de l'écotype américain dans les conditions écologiques françaises. On utilise des nichoirs de bois démon- 
tables selon les techniques américaines. On teste le bois de Pin (Pinus sp.) et deux bois africains : le Sipo (Entandrophragma) et le Framiré (Terminalia). Dans la majorité des élevages on laisse nidifier les femelles de première génération seulement. Puis on évalue les pertes subies par la progéniture de façon globale, et en fonction du lieu, de la période de ponte et du bois du nichoir. Les pertes globales varient de 50 à $95 \%$ des cellules. La mortalité due aux ennemis naturels indigènes (surtout Trichodes apiarius) affecte 10 à $50 \%$ des cellules construites. Des appâts polliniques sont efficaces contre Trichodes. La mortalité due à des causes indéterminées concerne 3 à $35 \%$ des cellules, et les émergences de deuxième génération 11 à $64 \%$. Les bois africains sont défavorables à la survie des stades immatures et accroissent le taux d'individus en diapause. Le Pin a des effets opposés. Si la deuxième génération nidifie, les émergences de la troisième génération sont peu nombreuses $(6 \%)$. Le climat ne permet pas une très grande activité et le meilleur rapport de multiplication apparente est 1,4 . Dans de bonnes conditions de sauvegarde on peut envisager l'élevage du mégachile dans les régions chaudes de France.

\section{INTRODUCTION}

L'abeille américaine solitaire et coupeuse de feuilles, Megachile pacifica Pz. ${ }^{1}$ longtemps nommée à tort par les auteurs Megachile rotundata F. est en fait une espèce paléarctique importée accidentellement aux U.S.A. vers les années 1930, probablement de l'Europe du Sud-Est (BoharT, 1970). M. pacifica se rencontre dans toute l'Europe de l'Espagne à la Finlande (Asensio de la Sierra et Rodriguez Ibanez, 1972 et Erlandsson, 1960). Petersen d'après Holm et Skou (1972) reconnaît que les exemplaires de $M$. rotundata auct. provenant de différents endroits d'Europe sont identiques au $M$. pacifica américain. Moczar (1959) et Benedek (1968 et 1969) ainsi que Solinas et BIN (1965) mentionnent cette espèce dans la faune indigène de leur pays respectif la Hongrie et l'Italie. Par ailleurs Desmier de Chenon (communication personnelle) a capturé $M$. pacifica en France dans le Sud-Est et la région parisienne. Le biotype américain posséderait les mêmes caractéristiques morphologiques que le biotype tchèque et différerait du biotype iranien (Bohart, communication personnelle).

La première capture officielle de $M$. pacifica est faite en 1947 par Krombein (1948) dans l'état de Washingion. Pendant la décade suivante l'espèce se propage rapidement d'Est en Ouest gagnant les États de la côte pacifique. Le mégachile éveille rapidement l'attention des spécialistes de la pollinisation par sa présence sur les champs de luzerne, son activité de butinage et sa rapidité de multiplication. Dès 1960 des sites artificiels de nidification sont mis en place à proximité des champs par des producteurs de semence de luzerne de l'Idaho, l'Utah, l'Oregon et du Nevada. Depuis ce temps la domestication du mégachile américain ne fait que progresser et les notes techniques sur ce sujet ne manquent pas : Stephen (1962); Hobss (1965-67-73); Walstrom et Jones (1968); Wilson (1968); Johansen et al (1969); Johansen

1. D'après HuRd (1967) $M$. rotundata $F$. est le synonyme de $M$. centuncularis L. espèce n'appartenant pas au sous genre Eutricharea. ReBmanN (1967) estime que $M$. rotundata auct. est identique au $M$. (eutricharea) pacifica Pz. aussi j’adopte comme HoLm et SKov (1972) cette dernière dénomination. 
(1971); Johansen et Eves (1971); Erickson et Wilson (1972); Bohart (1972). Notons que cette abeille utilisée pour polliniser des champs de luzerne à graines peut aussi effectuer des croisements de plantes en enceinte fermée : Heinrichs (1967); Guy, Lecomte, Ecalle (1969); Szabo et Smith (1970); Aubury et Rogers (1971).

Depuis quelques années plusieurs pays d'Amérique et d'Europe (Chili, Canada, Hongrie, Espagne) importent des cocons de l'abeille américaine et adoptent les techniques d'élevage mises au point par les entomologistes des U.S.A. En France la première tentative d'élevage en plein champ se déroule vers 1962 dans les régions de Lusignan près de Poitiers et de Montpellier (Leconte, communication personnelle) mais les conditions climatiques exceptionnellement défavorables ne permettent pas aux expérimentateurs de tirer de conclusion.

Devant la stagnation des rendements grainiers de luzerne il paraît important de maîtriser un facteur indispensable de la production : les insectes pollinisateurs. Il est donc souhaitable de connaître les possibilités d'adaptation dans notre pays d'une espèce pollinisatrice domestiquée. Dans ce but j'entreprends depuis deux ans (1972-1973) en collaboration avec la F.N.A.M.S. ${ }^{1}$ une expérimentation sur des $M$. pacifica importés du Canada. Le travail consiste à observer l'activité des insectes, évaluer leur pouvoir de multiplication et l'incidence des facteurs de l'environnement sur l'activité des femelles et la survie de la progéniture. Par ailleurs on met en œuvre des techniques destinées à réduire les pertes de toute origine et favoriser l'accroissement annuel des populations.

\section{BIOLOGIE DE MEGACHILE PACIFICA}

Megachile pacifica est certainement l'abeille solitaire dont les mours sont les mieux connues. Plusieurs travaux de thèse ont paru ces dernières années sur son comportement et son écologie (Tirgari, 1963; Kukovica, 1966; Lee, 1967; Osgood, 1967; Gerber, 1969; Packer, 1971; Eves, 1973).

Je décris dans les lignes qui suivent les principaux caractères de la biologie de $M$. pacifica : en Hongrie la période de vol s'étend de juin à septembre (BENEDEK, 1968), en France de juillet à octobre (surtout août et septembre). Les mâles apparaissent quelques jours avant les femelles. L'accouplement a lieu dès que ces dernières sortent de leur cocon; il se produit plusieurs fois chez le mâle mais probablement une seule fois chez la femelle. Deux ou trois jours après, la nidification commence. Les femelles recherchent des cavités tubulaires de 4 à $5 \mathrm{~mm}$ de diamètre, orientées de préférence vers l'Est ou le

1. Fédération nationale des agriculteurs multiplicateurs de semences. 
Sud. Elles ont tendance à fonder leur nid près de l'endroit de leur naissance et près $\mathrm{du}$ nid de leurs congénères. Une même femelle peut selon certains, construire et approvisionner plusieurs nids simultanément. Les femelles tapissent les parois de leur cavité de nidification de morceaux de feuilles oblongs qu'elles découpent grâce à leurs mandibules sur des espèces végétales diverses : Medicago sativa, Melilotus sp., Polygonum aviculare, Aster sp., Delphinium sp., Rosa sp.,... Des pétales d'Helianthus annuus sont aussi parfois utilisés pour construire les nids. Après la pose des morceaux de feuilles ou de pétales qui constituent la partie superficielle d'une cellule, les femelles accumulent des réserves de pollen et de nectar jusqu'à remplir leur petit pot aux deux tiers, puis elles déposent un œuf et ferment l'entrée avec plusieurs morceaux de feuille ronds. La base de la cellule suivante s'applique contre ce couvercle et ainsi de suite. Une cellule mesure environ $1 \mathrm{~cm}$ de long. Les nids, contenant de 1 à 10 ou 15 cellules, ont un aspect de cigare; ils possèdent un opercule ou bouchon terminal composé d'un grand nombre de découpures de feuilles rondes appliquées les unes contre les autres (Photos Pl. 1).

Chaque femelle pond en moyenne 25 à 30 œufs au cours d'une vie d'un à deux mois. Certains œufs sont fécondés et donnent des femelles, les autres ne le sont pas et donnent des mâles haplö̈des. Selon Krostermeyer et Soo (1968) les mâles ont 16 chromosomes et les femelles 32. La proportion des sexes varie en fonction du diamètre et de la longueur des cavités de nidification, mais on peut estimer que la proportion des femelles oscille en général entre $1 / 4$ et $1 / 3$ de 1 'effectif.

Les femelles butinent sur diverses plantes : Trifolium, Hedysarum, Melilotus, Medicago, Cichorium, Cosmos, Polygonum, Veronica, Mentha, Salvia, Lythrum, Galega, Eriogonum, Phacelia (DALY, 1952; Hund, 1954; Osgood, 1968). Cependant l'attirance des femelles est très grande pour les fleurs de papilionacées : Melilot et particulièrement Luzerne dont elles pollinisent les fleurs à une cadence de 11 à 15 fleurs déclenchées par minute. Le rayon d'action des femelles est très faible $(10$ à $40 \mathrm{~m}$ ) lorsque les fleurs sont abondantes dans les environs de leur nid, mais il peut atteindre $1500 \mathrm{~m}$ en cas de pénurie. L'activité de ces mégachiles est étroitement liée à la température de l'air et à l'ensoleillement. La température seuil est selon la plupart des auteurs $24-25{ }^{\circ} \mathrm{C}$.

A l'intérieur de sa cellule l'œuf éclot au bout de 2 à 3 jours et la larve mue 3 fois au cours de son repos. Après 3 semaines la larve de quatrième stade termine sa provision de pollen, rejette ses excréments au sommet de la cellule, construit un cocon brunâtre et s'immobilise comme toutes les prénymphes d'apoïdes (Photos Pl. 2). A partir de ce moment deux évolutions sont possibles : soit la prénymphe se transforme rapidement en nymphe puis en adulte pour donner une seconde génération annuelle, soit elle n'achève pas son développement et ne se transforme en nymphe que l'été suivant. La seconde géné- 
ration (et parfois la troisième) n'est jamais que partielle quelque soit l'environnement. Dans certaines conditions on observe une seule génération. HoLm et Skou (1972) estiment qu'à la sortie de l'hiver le $M$. pacifica américain a de plus gros besoins de chaleur pour donner des adultes, que les mégachiles communs au Danemark (M. centuncularis, M. willugbiella, M. leachella). L'importance de ces besoins est fonction de la longueur de la période d'hibernation (KRUNIC et Hinks 1972). Ces mêmes auteurs constatent l'inaptitude du $M$. pacifica américiain à hiberner dans l'Alberta (Canada) alors que le $M$. pacifica du Nord de l'Europe hiberne en Finlande. Cette observation permet de penser que chaque écotype a une physiologie propre, adaptée à un cycle saisonnier bien défini.

\section{MATÉRIEL ET MÉTHODES}

Quatre champs de production de semence de luzerne sont choisis comme champs expérimentaux dans le Sud-Est de la France en 1972 et 1973 . Un de ces champs se trouve à Sérignan près de la ville d'Orange dans le Vaucluse, les autres sont à Manosque et Greoux dans les Alpes de Haute-Provence.

\section{I. - Caractéristiques climatiques des périodes et des lieux d'expérience}

Les moyennes hebdomadaires des températures maxima et minima, ainsi que la pluviométrie pendant les périodes choisies pour la nidification des mégachiles sont inscrites dans le tableau 1. Le poste de météorologie de Sérignan se trouve à Orange. On remarque que les maxima et minima absolus $36^{\circ} \mathrm{C}$ et $5{ }^{\circ} \mathrm{C}$ sont enregistrés à Greoux. D'une façon générale l'année 1973 est plus chaude que l'année 1972. La région de Manosque et Gréoux aurait tendance à être plus chaude pendant le jour et plus froide pendant la nuit, que celle de Sérignan.

\section{II. - Importation, vérification de l'état sanitaire,}

hibernation et réactivation des cellules de mégachiles

Chaque année 12000 cocons de mégachiles sont importés du Canada. Au cours de l'hiver on découpe aux ciseaux un petit morceau de cocon dans la partie apicale de celui-ci, afin d'examiner le contenu. Après élimination des prénymphes mortes ou parasitées on conserve les prénymphes saines à une température de $5{ }^{\circ} \mathrm{C}($ H.R. $=70 \%)$. La date du lâcher des insectes est prévue pour la dernière semaine du mois de juin, a fin que les abeilles volent pendant la période la plus chaude. Cette dernière condition implique la pratique d'une précoupe retardant la floraison des champs de luzerne. On commence à réactiver les prénymphes 3 semaines avant le début de la floraison : les cocons sont placés dans des « éclosoirs " à une température de $20{ }^{\circ} \mathrm{C}$ pendant 48 heures, puis à la température de $32{ }^{\circ} \mathrm{C}(\mathrm{H} . \mathrm{R} .=50$ à $70 \%)$ jusqu'à ce que les premiers mâles apparaissent. A ce moment on transporte les “ éclosoirs 》 dans les abris à nichoirs installés dans les champs d'expérience.

\section{III. - Dispositif de nidification (Photos Pl. III)}

Le dispositif de nidification est inspiré du modèle recommandé par HoBbs (1973). Il comprend l'abri à nichoirs, les nichoirs et les “ éclosoirs ".

L'abri est une caisse en bois soit de $130 \times 110 \times 30 \mathrm{~cm}$ (grand modèle), soit de $70 \times$ $60 \times 15 \mathrm{~cm}$ (petit modèle). Il peut posséder ou non un auvent. L'ouverture est orientée vers l'Est. Deux piquets supportent la caisse et chacun d'eux est muni de plaques métalliques horizontales de $20 \mathrm{~cm}$ de diamètre a fin d'interdire l'ascension aux rongeurs. De même, pour empêcher des invasions de fourmis et forficules on enduit de glue la face inférieure des plaques métalliques. Les abris de grand modèle possèdent un grillage à maille de $2 \times 2 \mathrm{~cm}$ pour éviter les dégats causés par les oiseaux.

Les nichoirs de bois $(50 \times 24 \times 8 \mathrm{~cm})$ sont accrochés au fond de l'abri. Ils possèdent 33 rangées de 16 trous horizontaux de $8 \mathrm{~cm}$ de profondeur et $6 \mathrm{~mm}$ de diamètre (dimensions les plus favorables à une forte proportion de femelles d'après STEPHEN et Osgood, 1965 a.). 


\section{PLANCHE I}

Adultes, nid et cocon de M. pacifica (Photos S. Carré)

a) M. pacifica mâle

c) Nid

1. vue d'ensemble,

2. détail du bouchon terminal operculant le nid,

3 . dernière cellule construite. b) M. pacifica femelle découpant un opercule sur une feuille de Melilotus.

d) cocon débarrassé de son enveloppe de morceaux de feuilles.

1. partie apicale, 2. excréments.

Trait blanc

$$
\text { ou noir }-=4 \mathrm{~mm}
$$

\section{TAFEL I}

Adulte, Nest und Kokon von Megachile pacifica (phot. S. CARRÉ)

a) M. pacifica $\widehat{\delta}$

c) Nest

1. Gesamtansicht

2. Einzelansicht d. Verschlusspropfens b) M. pacifica $९$, beim Ausschneiden eines Deckels aus einem Melilotusblatt.

d) Kokon, von seiner Hülle aus Blattstückchen befreit

1. Apikalende

2. Exkremente

3. d. zuletzt gebaute Zelle 

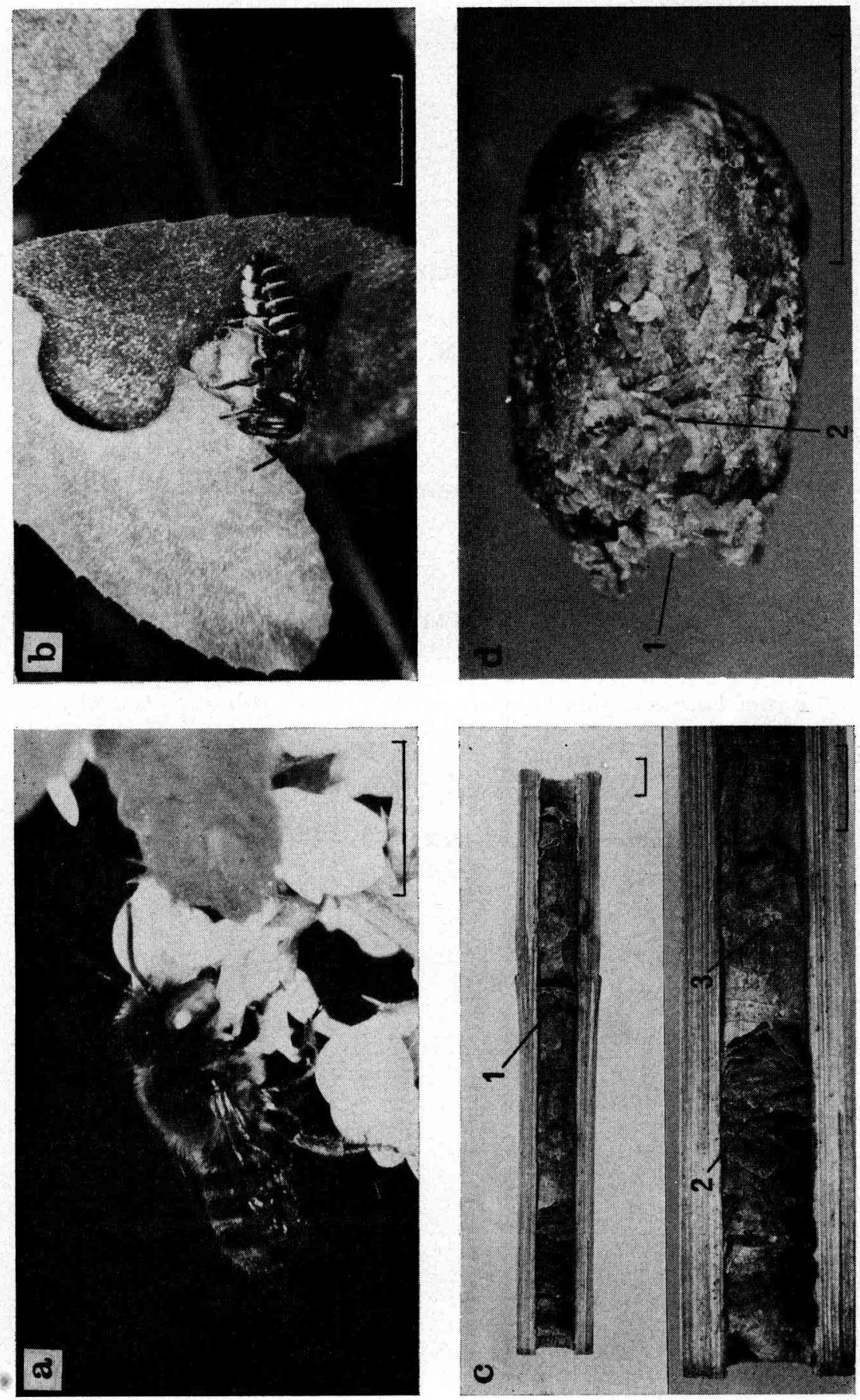


\section{PLANCHE II}

Stades de développement de M. pacifica (Photos S. Carré)
a) ouf
b) jeune larve
c) prénymphe
d) nymphe (tête)

Trait blanc ou noir $-=4 \mathrm{~mm}$

\section{TAFEL II}

Entwicklungsstadien von Megachile pacifica (phot. S. CarRÉ)
a) $\mathrm{Ei}$
b) Junge Larve
c) Pronymphe
d) Nymphe (Kopf)

schwarzer oder weisser Strich $-4 \mathrm{~mm}$ 

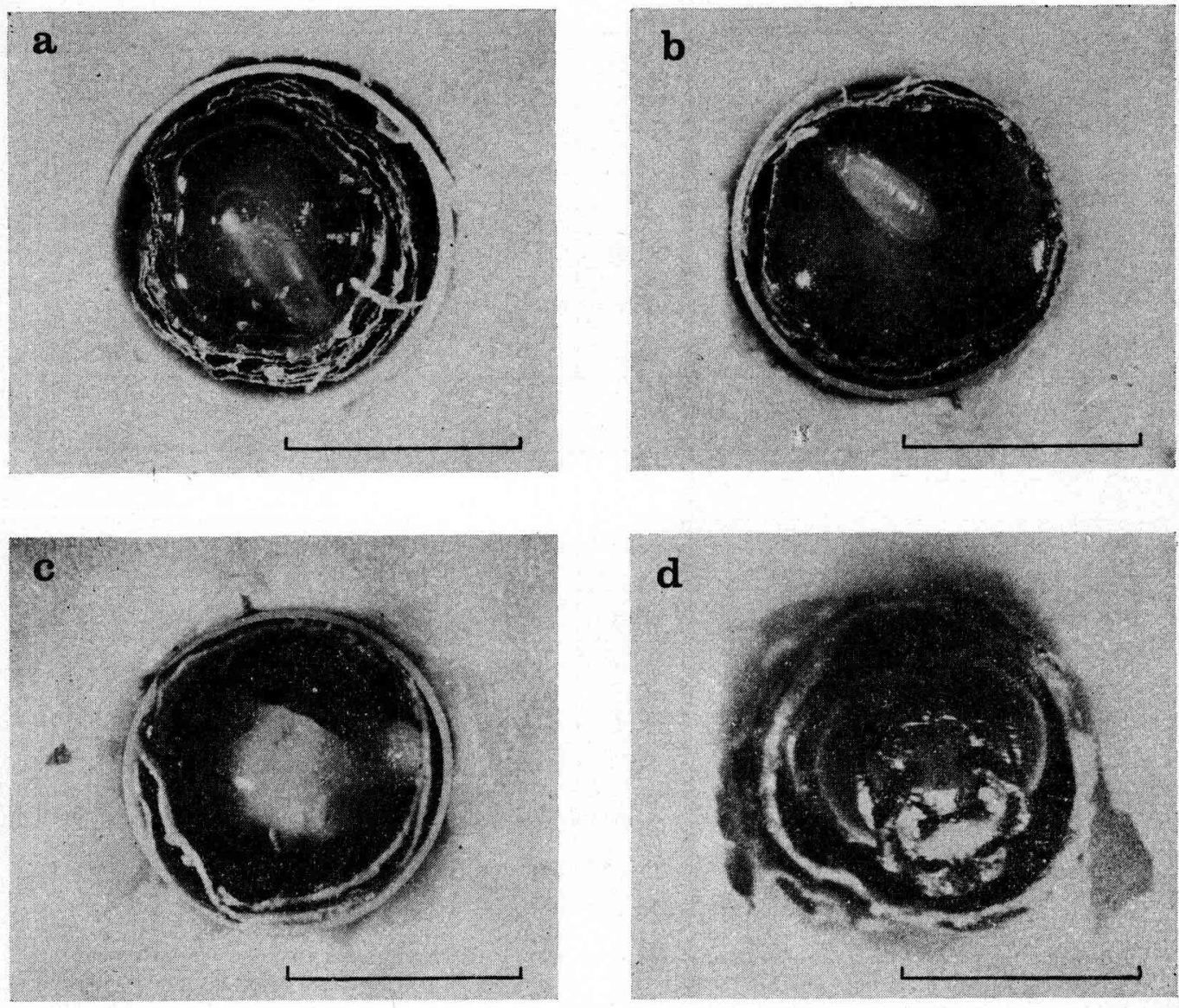
TABL. 1. - Température et pluviométrie pendant les périodes de nidification de Megachile pacifica

TABL. 1. - Temperaturen u. Niederschläge während der Nestbauperioden von Megachile pacifica

\begin{tabular}{|c|c|c|c|c|c|c|}
\hline 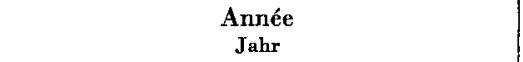 & \multicolumn{6}{|c|}{1972} \\
\hline $\begin{array}{c}\text { Lieu } \\
\text { Ort }\end{array}$ & \multicolumn{3}{|c|}{ Gréoux } & \multicolumn{3}{|c|}{ Sérignan } \\
\hline $\begin{array}{l}0 \text { - Pluie } \\
\text { Regenmenge }^{1}\end{array}$ & Max. & Min. & pluie & Max. & Min. & pluie \\
\hline $\begin{array}{l}\text { Du } 26 / 6 \text { au } 3 / 7 \ldots \ldots \cdots \cdots \cdots \cdots \cdots \cdots \cdots \\
\text { Vom 26.6. }-3.7 .\end{array}$ & 26,5 & 10,2 & 3 & 25,5 & 14,7 & 18 \\
\hline $\begin{array}{l}\text { Du } 4 / 7 \text { au } 11 / 7 \ldots \ldots \ldots \ldots \ldots \ldots \ldots \ldots \ldots \\
\text { von bis }\end{array}$ & 29,7 & 12,2 & 2 & 28,3 & 15,9 & 1 \\
\hline Du $12 / 7$ au $19 / 7 \ldots \ldots \ldots \ldots \ldots \ldots$ & 29,5 & 11,4 & 58 & 28,6 & 15,4 & 26 \\
\hline Du $20 / 7$ au $27 / 7 \ldots$ & 30,8 & 13,1 & $\mathbf{0}$ & 30,0 & 19,1 & 0,5 \\
\hline Du $28 / 7$ au $4 / 8 \ldots \ldots \ldots \ldots \ldots \ldots \ldots$ & 26,1 & 8,3 & 0 & 26,1 & 14,9 & 0 \\
\hline Du $5 / 8$ au $12 / 8 \ldots$ & 30,9 & 11,3 & 0 & 31,2 & 15,5 & 0 \\
\hline $\begin{array}{l}\text { Année } \\
\text { Jahr }\end{array}$ & \multicolumn{6}{|c|}{1973} \\
\hline $\begin{array}{l}\text { Du } 26 / 6 \text { au } 3 / 7 \ldots \ldots \cdots \cdots \cdots \cdots \cdots \cdots \cdots \\
\text { Vom } 26.6 .-3.7 \text {. }\end{array}$ & 31,2 & 12,4 & 0 & 32,0 & 16,6 & 0,7 \\
\hline $\begin{array}{l}\text { Du } 4 / 7 \text { au } 11 / 7 \ldots \ldots \ldots \ldots \ldots \ldots \ldots \ldots \ldots \\
\text { von bis }\end{array}$ & 31,5 & 10,9 & 0 & 31,6 & 18,3 & $\mathbf{0}$ \\
\hline Du $12 / 7$ au $19 / 7 \ldots \ldots \ldots \ldots \ldots \ldots \ldots$ & 29,1 & 12,0 & 12 & 28,5 & 16,5 & 39,9 \\
\hline Du $20 / 7$ au $27 / 7 \ldots$ & 27,1 & 10,2 & $\mathbf{0}$ & 26,1 & 15,9 & 22,6 \\
\hline$\ldots \ldots \ldots \ldots \ldots$ & 29,1 & 11,5 & 0 & 27,7 & 15,6 & 0 \\
\hline Du $5 / 8$ au $12 / 8$ & 32,0 & 12,7 & 0 & 32,1 & 16,7 & $\mathbf{0}$ \\
\hline
\end{tabular}

1. Moyennes des températures maxima et minima journalières en degrés C. Précipitations en mm pendant les périodes indiquées. Zeit.

1. Mittel der täglichen Höchst- $\mathbf{u}$. Tiefsttemperaturen in Celsiusgraden. Niederschläge in mm während der angegebenen

Ces nichoirs construits avec des plaques de bois cannelées empilées les unes sur les autres sont démontables, ce qui rend possible l'extraction des cellules sans dommages. Sur leur face percée sont peintes en noir des lettres qui recouvrent 39 à $46 \%$ de la surface totale. Leur dessin permet aux abeilles de retrouver plus facilement l'emplacement de leur nid. Trois sortes de bois sont utilisées : un bois européen : le Pin ${ }^{1}$ et deux bois africains : le Sipo ${ }^{2}$ et le Framiré ${ }^{3}$.

Les c éclosoirs m sont des casiers de $40 \times 12 \times 6 \mathrm{~cm}$ que l'on place dans l'abri au-dessus des nichoirs. Ils ont un couvercle grillagé et un de leur côté possède une rangée de 5 trous de $1 \mathrm{~cm}$ de diamètre permettant la sortie des adultes. L'épaisseur de la couche de cocons à l'intérieur d'un éclosoir ne dépasse pas $1 \mathrm{~cm}$.

En 1973 tous les abris sont protégés par un brise-vent de $10 \times 2,5 \mathrm{~m}$. Un seul abri renferme 2 petits nichoirs destinés à piéger des ennemis des mégachiles (Trichodes apiarius L. :

1. Pinus silvestris L. ou $P$. pinaster Soland (Abietacée).

2. Entandrophragma utile Sprague (Meliacée).

3. Terminalia irorensis A. Cher. (Combretacée). 
coléoptère Cleridae). Ces nichoirs de $15 \times 12 \times 8 \mathrm{~cm}$ sont en Pin et sont construits sur le même principe que ceux qui sont destinés aux mégachiles. Ils sont percés de trous de 3 diamètres différents : 4-6 et $7 \mathrm{~mm}$. Par ailleurs au fond de chaque tube se trouve une provision pâteuse de pollen (pelotes d'abeilles domestiques) mélangé à de l'eau sucrée devant jouer le rôle d'appât pour les femelles de Trichodes.

\section{IV. - Observations sur le champ et en laboratoire}

Après le lâcher l'observateur humidifie tous les deux jours les cocons dans les éclosoirs. Ce traitement poursuivi pendant 8 à 10 jours doit éviter le dessèchement des nymphes dont

TABL. 2. - Conditions expérimentales des élevages

TABL. 2. - Versuchsbedingungen der Aufzuchten

\begin{tabular}{|c|c|c|c|c|c|c|}
\hline & $\begin{array}{c}\text { Année } \\
\text { Jahr }\end{array}$ & 19 & & & 1973 & \\
\hline & $\begin{array}{c}\text { Lieu } \\
\text { Ort }\end{array}$ & Manosque & Sérignan & Gréoux & Manosque & Sérignan \\
\hline 宓 & $\underset{\text { Anzahl }}{\text { Nombre }} \ldots \ldots \ldots \ldots \ldots$ & 5000 & 6000 & 4970 & 1750 & 5370 \\
\hline 产 & $\underset{\text { Urigine }}{\text { Origing }} \cdots \ldots \ldots \ldots \ldots$ & Canada & Canada & Canada & $\begin{array}{c}\text { Élevage } 1972 \\
\text { (Manosque } \\
\text { Sérignan) }\end{array}$ & Canada \\
\hline 象总 & $\underset{\text { Anzahl }}{\text { Nombre }} \ldots \ldots \ldots \ldots \ldots$ & 5 & 5 & 5 & 2 & 5 \\
\hline 章离 & $\underset{\text { Holzart }}{\text { Bois }} \ldots \ldots \ldots \ldots \ldots \ldots$ & $\begin{array}{l}\text { Pin }=1 \\
\text { Sipo }=2 \\
\text { Framiré }=2\end{array}$ & $\begin{array}{l}\text { Pin }=3 \\
\text { Sipo }=1 \\
\text { Framiré =1 }\end{array}$ & $\begin{array}{l}\text { Pin }=1 \\
\text { Sipo }=2 \\
\text { Framiré }=2\end{array}$ & $\begin{array}{l}\text { Pin }=1 \\
\text { Framiré }=1\end{array}$ & $\begin{array}{l}\text { Pin }=3 \\
\text { Sipo }=1 \\
\text { Framiré }=1\end{array}$ \\
\hline $\begin{array}{l}\text { Abri p } \\
\text { Schutzr }\end{array}$ & $\begin{array}{l}\text { ur nichoirs } \ldots \ldots \ldots \ldots \cdots \\
\text { um für d. Nistanlagen }\end{array}$ & $\begin{array}{l}\text { grand } \\
\text { gross }\end{array}$ & $\begin{array}{c}\text { grand } \\
\text { gross }\end{array}$ & grand & petit & grand \\
\hline $\begin{array}{l}\text { Auven } \\
\text { Schutzd }\end{array}$ & $\ldots \ldots \ldots \ldots \ldots$ & + & + & 0 & 0 & 0 \\
\hline $\begin{array}{l}\text { Brise } \\
\text { Windsel }\end{array}$ & irm $2,4 \times 10 \ldots \ldots \ldots \ldots$ & 0 & 0 & + & + & + \\
\hline $\begin{array}{l}\text { Piège } \\
\text { Trichod }\end{array}$ & $\underset{\text { Trichodes }}{\text { T.Falle }}$. & 0 & 0 & 0 & 0 & + \\
\hline 름 & $\begin{array}{l}\text { Pose des nichoirs } \ldots \ldots \ldots \\
\text { Anbringen d. Nistanlagen }\end{array}$ & 5 juillet & 19 juin & 27 juin & 27 juin & 26 juin \\
\hline$\tilde{\theta}$ & $\begin{array}{l}\text { Retrait des nichoirs ..... } \\
\text { Entfernen d. Nistanlagen }\end{array}$ & 12 août & 13 août & 27 juillet & 17 août & 12 septembre \\
\hline $\begin{array}{l}\text { Nombi } \\
\text { Zahl d. }\end{array}$ & $\begin{array}{l}\text { e de générations nidifiant } \\
\text { auenden Generationen }\end{array}$ & 1 & 1 & 1 & 1 & 2 \\
\hline $\begin{array}{l}\text { Variét } \\
\text { Luzerne }\end{array}$ & de luzerne $\ldots \ldots \ldots \ldots$. & Verneuil & Elga & Provence & Verneuil & Elga \\
\hline $\begin{array}{l}\text { Stocka } \\
\text { avant } \\
\text { Aufbew } \\
23^{\circ} \mathrm{C} \text { vo }\end{array}$ & $\begin{array}{l}\text { ge de la progéniture à } 20-23 \text { o } \\
\text { ibernation } \ldots \ldots \ldots \ldots \ldots \ldots \ldots \\
\text { hren der Nachkommen bei } 20 \text { - } \\
\text { der Uberwinterung }\end{array}$ & + & + & $\begin{array}{l}\frac{1}{2} \text { à } 20-23^{\circ} \mathrm{C} \\
\frac{1}{2} \text { à } 19^{\circ} \mathrm{C}\end{array}$ & + & + \\
\hline
\end{tabular}




\section{PLANCHE III}

Dispositif de nidification pour M. pacifica (Photos S. CARrÉ)

a) Vue générale d'un abri

1. éclosoir

2. nichoir

3. piège à Trichodes

4. brise-vent b) Détail d'un nichoir et d'un éclosoir

1. nichoir

2. plaque de bois cannelée du nichoir

3. “ éclosoir ) grillagé sur la partie supérieure

4. trou de sortie

TAFEL III

Vorrichtung für Nistanlagen von Megachile pacifica (phot. S. CARRÉ)

a) Gesamtansicht eines

Schutzraumes

1. “Schlüpfraum )

2. Nestanlage

3. Falle für Trichodes

4. Windschutz b) Einzelansicht einer

Nistanlage $u$. eines

(Schlüpfraumes »

1. Nistanlage

2. geriefte Holzplatte

d. Nistanlage

3. oben vergitterter (C Schlüpfraum )

4. Ausgangsöffnung 

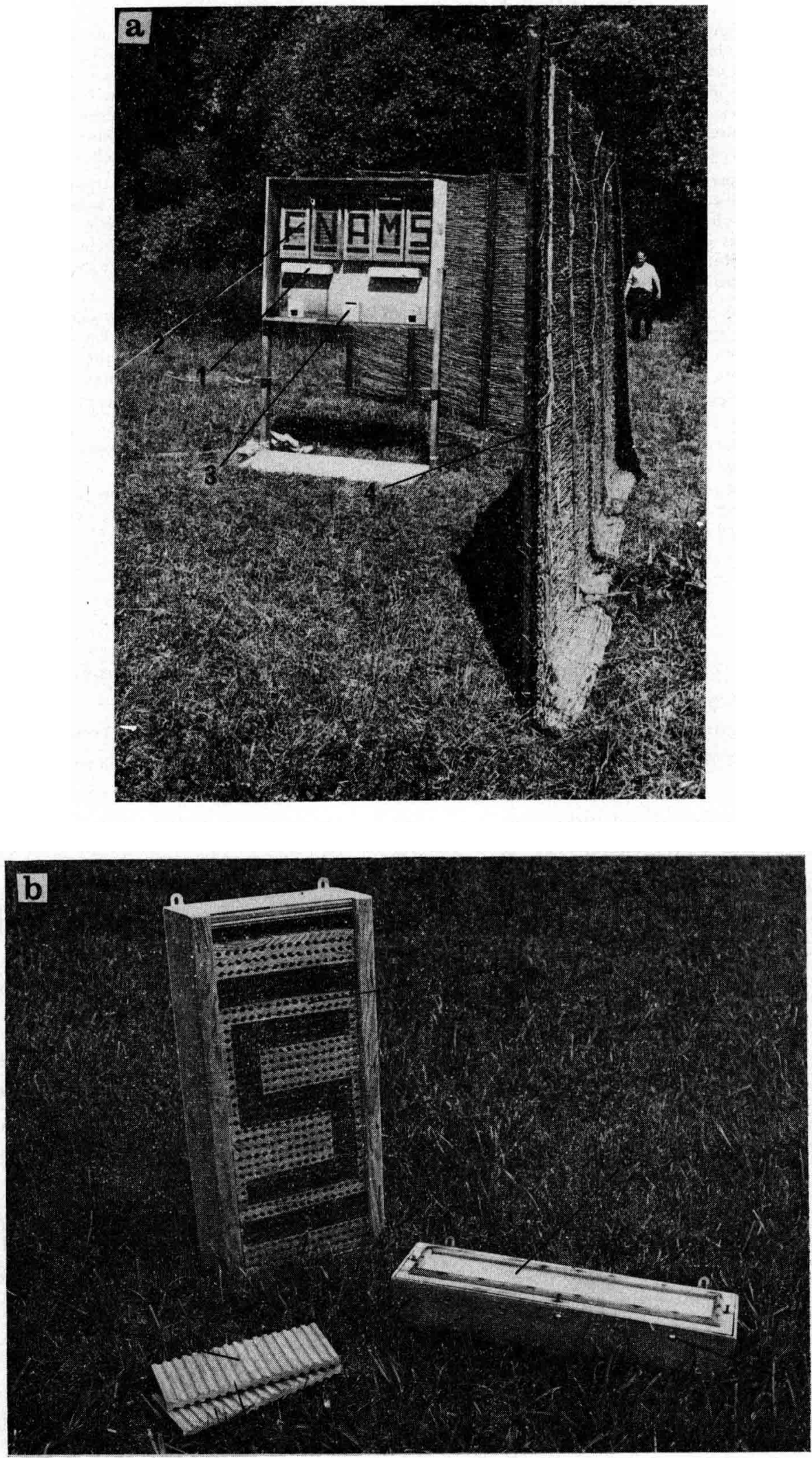
le développement est retardé (surtout chez les femelles). Par ailleurs deux fois par semaine on compte le nombre de nids operculés en relevant leur emplacement sur les nichoirs. Au bout d'un mois on repère la sortie des premiers individus émergeant en deuxième génération, qui fracturent les bouchons terminaux construits par leur mère. A ce moment là, soit on enlève les nichoirs avant le début des premières constructions de la deuxième génération, soit on laisse les nichoirs un mois de plus pour permettre la nidification de la deuxième génération. Tout au long de la nidification on évalue dans chaque nichoir le nombre de femelles en activité.

Lorsque la période de nidification est terminée on range les nichoirs à la température du laboratoire $\left(20-23^{\circ} \mathrm{C}\right)$ ou bien en chambre climatisée $\left(19^{\circ} \mathrm{C}\right)$. Les nichoirs sont entourés d'une toile de nylon a fin de récupérer les adultes qui émergent, ainsi que les parasites. Quand les émergences sont achevées on commence à démonter les nichoirs et inspecter le contenu de chaque cellule. On trie de cette manière les cellules attaquées par les parasites et les prédateurs et celles qui renferment une prénymphe en diapause.

La conception des élevages est différente en 1972 et 1973 . Pour avoir une vue rapide des conditions expérimentales de tous les élevages on doit consulter le tableau 2. En 1972 on fait nidifier des insectes d'importation seulement pendant la première génération, dans deux champs, un à Manosque, l'autre à Sérignan. En 1973 on a trois types d'élevage :

a) à Sérignan, un lot d'abeilles importées la même année nidifie pendant 2 générations de juin à septembre,

b) à Gréoux, un lot d'abeilles importées la même année nidifie seulement pendant la première génération,

c) à Manosque on fait nidifier la première génération issue des élevages de 1972 . Il s'agit uniquement d'insectes ayant eu un repos larvaire estival puisque l'on élimine systématiquement en 1972 tous les insectes émergeant en deuxième génération.

\section{RÉSULTATS}

Les résultats sont exposés dans l'ordre des phases de l'expérience. La première phase est la réactivation pendant laquelle les prénymphes deviennent adultes sous l'effet de la température constante de l'étuve et des températures alternées dans le champ. La seconde phase est la nidification; la troisième est la préhibernation que l'on met à profit pour inspecter les nids, noter la mortalité et les sorties d'individus en deuxième (ou troisième) génération.

Lors de l'étude de chaque période on se rend compte que les mégachiles subissent des pertes plus ou moins élevées ou qu'ils manifestent une aptitude plus ou moins grande à se multiplier. C'est l'analyse des pertes et du comportement que j'expose dans les trois paragraphes suivants.

\section{I. - La réactivation}

La réactivation totale comprend deux périodes : la première se passe en étuve à $32{ }^{\circ} \mathrm{C}$, la seconde sur le champ pendant un temps variable qui dépend du climat et de la somme des températures de la phase en étuve. Le tableau 3 donne les taux de la mortalité survenue à l'intérieur des éclosoirs. Ils sousestiment les pertes réelles dues à une réactivation trop longue. En effet beaucoup de mâles et de femelles claustrées trop longtemps sans nourriture parviennent à sortir des éclosoirs au moment du lâcher puis meurent au pied de l'abri et ne peuvent plus être comptabilisées. On constate un niveau généralement élevé de pertes, pouvant atteindre $19 \%$. Les réactivations trop 
longues : supérieures à 640 degré-jour correspondent à des pertes élevées chez les adultes mâles à Gréoux et Sérignan en 1973. La mortalité aux stades prénymphe et nymphe atteint son maximum à Sérignan en 1972 et à Manosque en 1973. Un seul site : Manosque, 1972, a relativement peu de mortalité $(4,6 \%)$ c'est l'indice d'une bonne réactivation en étuve et dans le champ.

TABL. 3. - Mortalité en " éclosoir 》 après la réactivation complète des cellules

ТАв, 3. - Sterblichkeit im « Schlüpfraum » nach der vollständigen Reaktivierung der Zellen

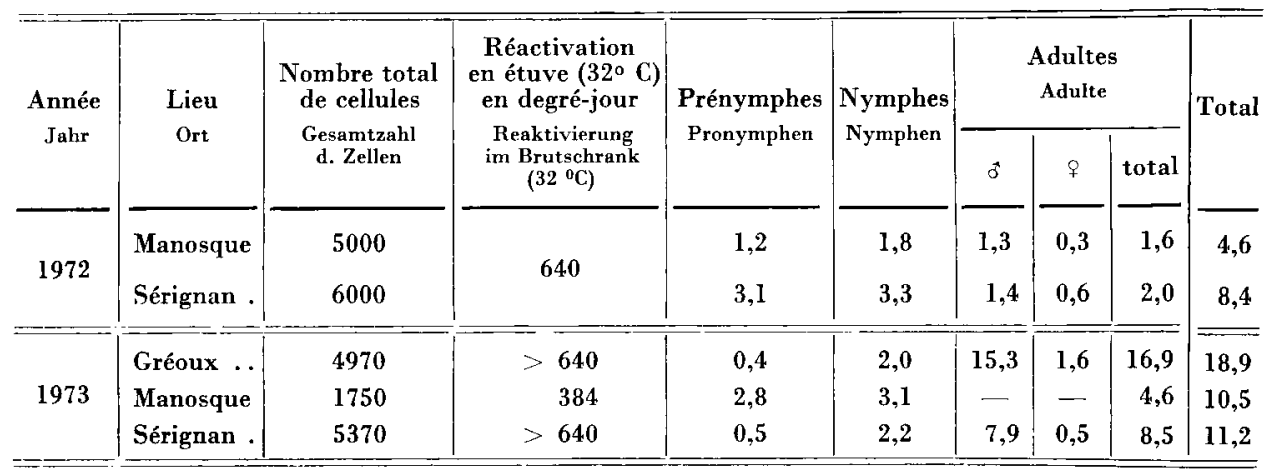

\section{II. - La nidification}

La durée de la nidification de la première génération de femelles est d'environ un mois quelque soit le lieu et l'année. La deuxième génération commence à émerger dès que la première n'est plus en activité (fig. 1-B). Le développement complet et l'émergence des premiers mâles nécessite environ 680 degré-jour. Les différences d'allure des courbes de la figure 1 sont dues au type de réactivation qui peut soit échelonner soit regrouper dans le temps les sorties des femelles. Les aléas climatiques, vent et baisse de température, sont aussi responsables des irrégularités plus ou moins prononcées des courbes de nidification.

\section{A - Mode d'occupation des nichoirs}

\section{1) Occupation des tubes}

Les femelles profitent plus ou moins complètement des tubes de nidification qui leur sont offerts en construisant de 1 à 8 cellules par tube. La moyenne du nombre de cellules par tube varie selon le nichoir entre 4 et 5 . Les bouchons terminaux peuvent être appliqués à l'extrémité de cavités ne renfermant aucune cellule, par contre ils font défaut à certains nids. Le tableau 4 donne les pourcentages des nids operculés pour chaque nichoir. La variabilité de cette donnée est assez forte puisque les pourcentages s'échelonnent de $46 \%$ à $85 \%$. 
TABL. 4. - Operculation des nids par les femelles en fonction du lieu et du bois du nichoir TaB. 4. - Verdeckeln d. Nester durch die Weibchen in Abhängigkeit von Ort und Holzart der Nistanlage

\begin{tabular}{|c|c|c|c|c|c|c|c|}
\hline \multirow{2}{*}{$\begin{array}{c}\text { Année } \\
\text { Jahr }\end{array}$} & Holz & \multicolumn{2}{|c|}{ Pin } & \multicolumn{2}{|c|}{ Sipo } & \multicolumn{2}{|c|}{ Framiré } \\
\hline & $\begin{array}{l}\text { Lieu } \\
\text { Ort }\end{array}$ & $\%^{1}$ & $N^{2}$ & $\%$ & $\mathbf{N}$ & $\%$ & $\mathbf{N}$ \\
\hline \multirow{5}{*}{1972} & Manosque .. & 76,1 & 284 & 60,6 & 317 & 69,7 & 330 \\
\hline & & & & 64,0 & 361 & 66,1 & 298 \\
\hline & & 69,6 & 56 & & & & \\
\hline & Sérignan ....... & 70,2 & 245 & 46,1 & 258 & 60,2 & 241 \\
\hline & & 90,9 & 22 & & & & \\
\hline \multirow{3}{*}{1973} & Gréoux ... & 81,6 & 179 & & & & \\
\hline & Manosque..... & & & & & 84,6 & 408 \\
\hline & Sérignan .... & 82,9 & 463 & & & & \\
\hline \multicolumn{3}{|c|}{$\begin{array}{l}\text { 1. Pourcentage de nids operculés. } \\
2 \text {. Nombre total de nids construits. }\end{array}$} & \multicolumn{5}{|c|}{$\begin{array}{l}\text { 1. \% der verdeckelten Nester. } \\
\text { 2. Gesamtzahl der gebauten Nester. }\end{array}$} \\
\hline
\end{tabular}

2) Attractivité des différents bois - Phénomène de grégarisme

La figure 2 montre qu'en 1972 les femelles se répartissent dans tous les nichoirs dès le début de la période de nidification, quelque soit le bois. A Manosque on remarque au début une préférence assez légère pour le nichoir de Pin. A Sérignan on assiste à un regroupement des femelles dans les trois nichoirs centraux. En 1973, dans chacun des trois élevages, un seul nichoir est occupé. De ces observations on ne peut pas conclure à une attirance pour un bois comme on aurait pu s'y attendre, mais plutôt à un grégarisme assez évident. Ce phénomène ne se manifeste pas à Manosque (1972). Il est faible à Sérignan (1972) et très fort dans les trois élevages de 1973.

L'interattraction individuelle semble être inversement proportionnelle au nombre de femelles par unité de surface de nichoir. 60 femelles par nichoir est probablement le seuil au-delà duquel les effets de l'interattraction cessent d'être visibles. En deçà, la tendance au regroupement s'exprime parfois d'une manière éclatante comme dans l'élevage de Sérignan (1973) où les deux générations ont dédaigné 4 nichoirs sur 5 pour peupler très abondamment le nichoir central (463 nids construits dans un nichoir offrant 528 tubes de nidification). 


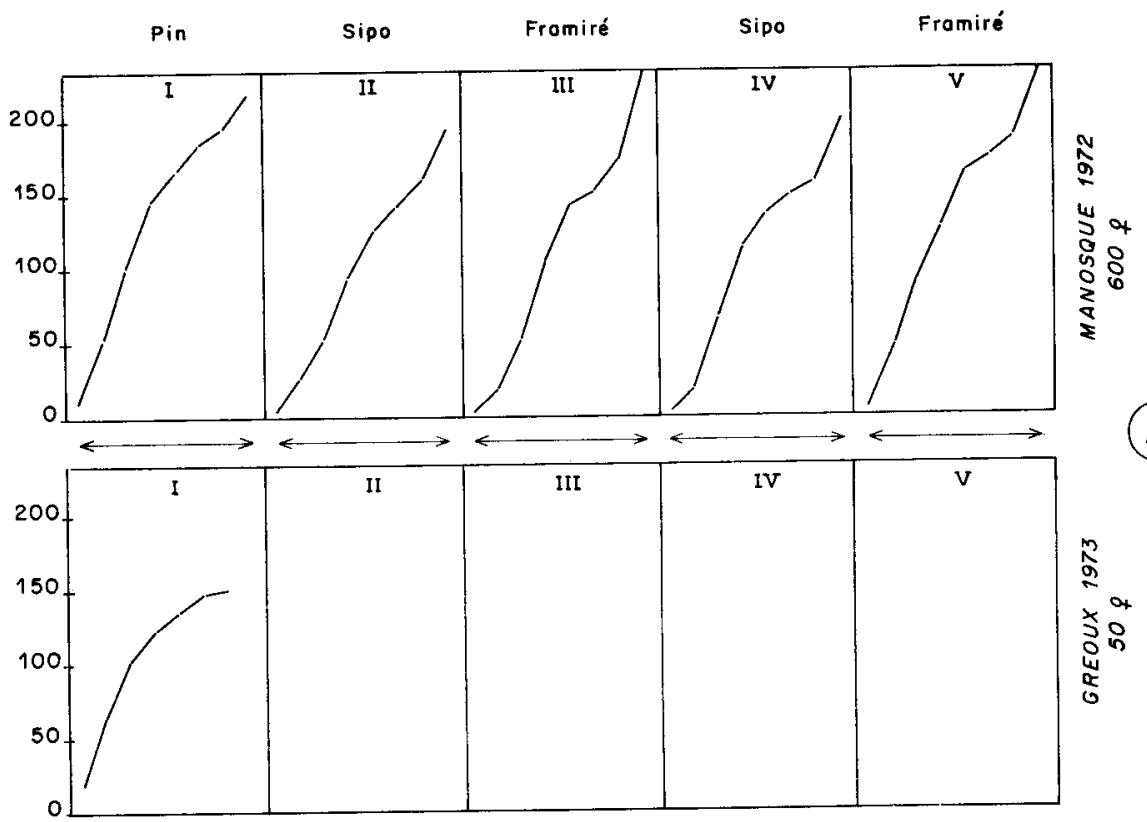

(A)

2

$\Varangle 0$

造

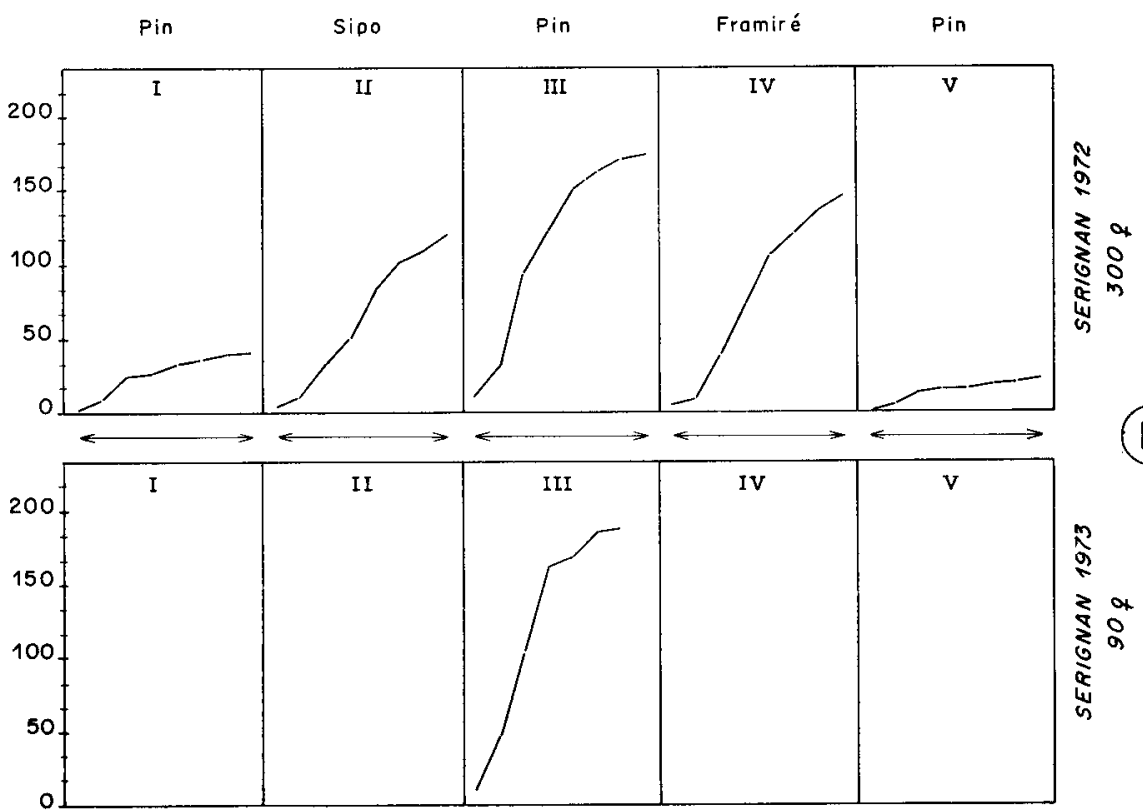

(B) 


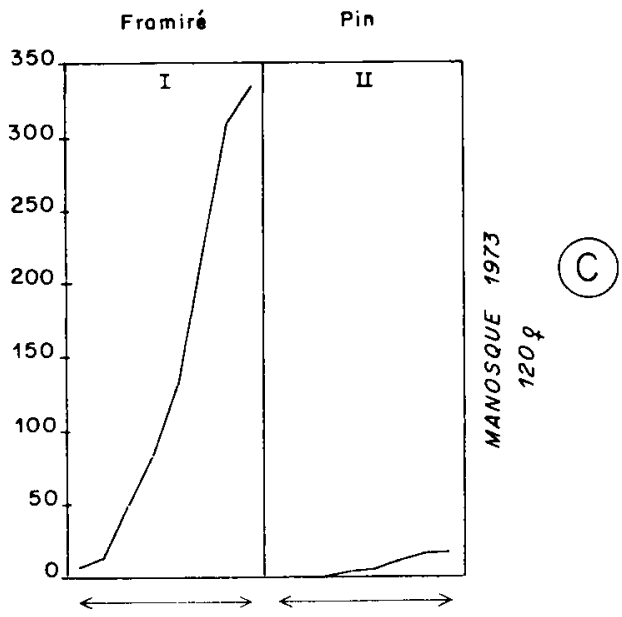

Fig. 2. - Mode d'occupation des nichoirs et grégarisme au cours de la $1^{\mathrm{re}}$ génération annuelle de Megachile pacifica

Verticalement $=$ Nombre total de nids operculés

Horizontalement $=$ Période de nidification d'un mois environ $\longleftrightarrow$

$\mathbf{A}=$ Grand abri à 5 nichoirs

$\mathrm{B}=$ Grand abri à 5 nichoirs

$\mathrm{C}=$ Petit abri à 2 nichoirs

I à $\mathrm{V}=$ Ordre de rangement des nichoirs de gauche à droite dans l'abri.

Anu. 2. - Art der Annahme von Nistanlagen und Gregarismus im Verlauf der 1. Generation eines Jahres von Megachile pacifica

Ordinate $=$ Gesamtzahl der verdeckelten Zellen

Abszisse $=$ Nestbauperiode während eines Monats etwa $\longleftrightarrow$

$A=$ grosser Schutzraum mit 5 Nistanlagen

$\mathbf{B}=$ desgl.

$\mathrm{C}=$ kleiner Schutzraum mit 2 Nistanlagen

I bis $\mathrm{V}=$ Reihenfolge der Nistanlagen von links nach rechts im Schutzraum

\section{3) Attractivité des différentes parties des nichoirs}

Les parties du nichoir peintes en noir sont préférées aux parties claires. La différence d'attractivité est bien visible lorsque peu de femelles nidifient (tableau 5). La partie basse du nichoir, plus longtemps exposée au soleil est plus attractive que la partie haute protégée des rayons par le toit de l'abri (tableau 6). Comme dans le cas précédent la disparité du peuplement entre la partie ombrée et la partie ensoleillée s'atténue lorsque le nombre de nids par nichoir augmente.

On peut dire que l'homogénéité de la répartition des nids dans un ensemble de nichoirs dépend de trois facteurs, par ordre d'importance :

- Le nombre de femelles par unité de surface de nidification;

- L'égalité de l'ensoleillement de la surface des nichoirs;

- La présence de dessins de couleur noire sur le bois. 
TABL. 5. - Attractivité des trous situés dans les parties des nichoirs peintes en noir, en 1972

TABL. 5. - Anziehungskraft der Löcher in den schwarz gestrichenen Teilen der Nistanlagen (1972)

\begin{tabular}{|c|c|c|c|c|c|c|c|c|c|c|}
\hline $\begin{array}{c}\text { Lieu } \\
\text { Ort. }\end{array}$ & \multicolumn{5}{|c|}{ Manosque } & \multicolumn{5}{|c|}{ Sérignan } \\
\hline $\begin{array}{l}\text { Nichoir }{ }^{\mathbf{1}} \\
\text { Nistanlage }\end{array}$ & F & $\mathbf{N}$ & A & $\mathbf{M}$ & $S$ & $\mathrm{~F}$ & $\mathbf{N}$ & $\mathbf{A}$ & $\mathbf{M}$ & $\mathbf{S}$ \\
\hline $\begin{array}{l}\text { Nbre total de nids construits. } \\
\text { Gesamtzahl d. gebauten Nester }\end{array}$ & 284 & 317 & 298 & 359 & 330 & 56 & 258 & 245 & 241 & 22 \\
\hline $\begin{array}{l}\% \text { de nids }{ }^{2} \quad \ldots \ldots \ldots \ldots \cdots \\
\% \text { d. Nester }\end{array}$ & 52,1 & 54,4 & 51,2 & 52,4 & 56,1 & 82,3 & 53,7 & 53,6 & 55,7 & 96,2 \\
\hline
\end{tabular}

1. Lettre peinte en noir sur chacun des nichoirs.

2. Pourcentage de nids construits dans les trous situés dans les parties peintes en noir (Après pondération des nombres par le rapport de la surface blanche à la surface peinte).

1. Schwarzer Buchstabe auf jeder Nistanlage.

2. Prozentsatz der in den schwarz gestrichenen Teilen gebauten Nester (Nach Schätzen der Zahlen hinsichtlich weisser und gestrichener Oberfläche).

TABL. 6. - Attractivité de la partie des nichoirs exposée au soleil

TARL. 6. - Anziehungshraft der der Sonne ausgesetzten Teile der Nistanlage

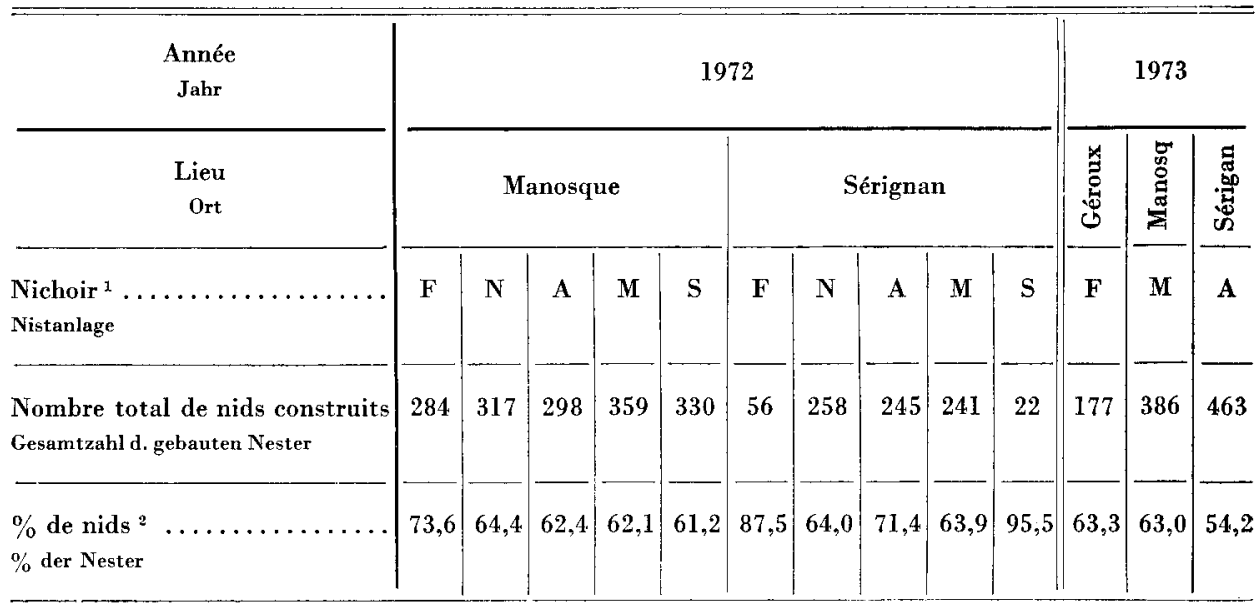

1. Même légende que pour le tableau 5 .

2. Pourcentage de nids construits dans la moitié inférieure, exposée au soleil, de chaque nichoir.

1. wie in Tabelle 5 .

2. \% der Nester, die in der unteren, der Sonneneinstrahlung ausgesetzten Hälfte jeder Nistanlage gebaut wurden.

\section{B - Pertes de femelles - Diminution de l'activité}

1) Estimation des pertes de femelles et de la fécondité

Dans les meilleurs élevages la fécondité moyenne est évaluée à 10 cellules par femelle en 1972 et 17 cellules par femelle en 1973. Par ailleurs si l'on 
compare le nombre de femelles en activité pendant la période de nidification intense, au nombre de femelles lâchées, on constate que 50 à $95 \%$ des effectifs disparaît.

\section{2) Facteurs en cause}

On ne possède aucun résultat concernant certains facteurs de pertes de femelles : maladies, parasitisme par acariens, prédation par les oiseaux ou les araignées, égarement hors $\mathrm{du}$ site artificiel de nidification. Par contre on a déjà vu plus haut qu'une mauvaise réactivation peut être la cause de pertes importantes d'adultes. On peut vérifier l'action néfaste du vent et des basses températures diurnes : Un vent de $15 \mathrm{~m} / \mathrm{s}$ à Sérignan (1972) cause de grosses pertes dans la population (fig. 1-A) Les températures comprises entre 20 et $25^{\circ} \mathrm{C}$ ne permettent pas une activité intense des femelles (fig. 1). Enfin il est bon de signaler qu'en 1972 et 1973 un assez grand nombre de vieilles femelles sont retrouvées parasitées et mortes dans les tubes des nichoirs. Elles ont un abdomen démesurément allongé à l'intérieur duquel se trouve une pupe de diptère Conopidae : Physocephala pusilla Meig (photo pl. 4). En 1972 près de 90 femelles parasitées sont récoltées à Manosque et 54 à Sérignan. En 1973, 123 femelles sont parasitées et sont mortes dans les trois abris. L'usure des ailes, et la rareté de la pilosité des femelles portent à croire que les mégachiles supportent l'endoparasite et nidifient comme les femelles saines. Les pupes sont très fortement parasitées par un hyménoptère Pteromalidae.

\section{C - Accroissement global de la population}

Si l'on fait abstraction de toutes les sortes de pertes aussi bien chez les adultes que chez la progéniture, on peut évaluer l'accroissement de la population d'une génération à l'autre en calculant le rapport :

Nombre de cellules ( fille ) construites dans les nichoirs

Nombre de cellules « parent » placées dans les éclosoirs

En fait il est préférable d'utiliser le rapport :

Nombre de femelles (c fille )

Nombre de femelles « parent »

qui donne une idée plus juste de l'activité des femelles. En effet la proportion des sexes peut varier de façon très sensible d'une population à l'autre (12 à $28 \%$ de femelles). La proportion des femelles est évaluée chez la population fille de la manière suivante : on calcule le pourcentage des femelles de la deuxième génération qui émerge en laboratoire; puis celui des femelles de la population en diapause, après hibernation et réactivation d'un lot de prénymphes. 
On estime que le pourcentage moyen obtenu est celui de l'ensemble de la population fille (y compris les cellules détruites).

Les résultats du tableau 7 montrent qu'il y a suivant les cas, soit une régression, soit un léger accroissement de la population. La régression la plus marquée s'observe en 1973, à Sérignan et surtout à Gréoux. Elle est due en grande partie à une mauvaise technique de réactivation en étuve. L'accroissement le plus fort est enregistré à Manosque en 1973 : (x 1,4). Un fait intéressant n'apparaît pas dans le tableau 7 . Il s'agit de l'accroissement du nombre

TABL. 7. - Possibilité d'accroissement de la population

TAB. 7. - Möglichkeit der Populationszunahme

\begin{tabular}{|c|c|c|c|c|c|c|}
\hline \multirow[t]{2}{*}{$\begin{array}{l}\text { Année } \\
\text { Jahr }\end{array}$} & \multirow[t]{2}{*}{$\begin{array}{c}\text { Lieu } \\
\text { Ort }\end{array}$} & \multicolumn{2}{|c|}{$\begin{array}{l}\text { Population parent (« éclosoir ») } \\
\text { "Elternpopulation (Schliüpfraum ") }\end{array}$} & \multicolumn{2}{|c|}{$\begin{array}{l}\text { Population fille ' (nichoirs) } \\
\text { Tochterpopulation (Nistanlagen) }\end{array}$} & \multirow{2}{*}{$\begin{array}{c}\begin{array}{c}\text { Coefficient } \\
\text { de multiplication } \\
\text { Vermehrungs- } \\
\text { koeffizient }\end{array} \\
\text { \& } \mathbf{F} / \text { P }^{2}\end{array}$} \\
\hline & & $\begin{array}{c}\text { Nombre total } \\
\text { de cellules } \\
\text { Gesamtzahl } \\
\text { der Zellen }\end{array}$ & 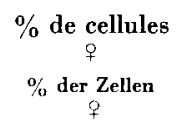 & $\begin{array}{l}\text { Nombre total } \\
\text { de cellules } \\
\text { Gesamtzahl } \\
\text { der Zellen }\end{array}$ & $\begin{array}{c}\% \text { de cellules } \\
q \\
\% \text { der Zellen } \\
q\end{array}$ & \\
\hline 1972 & $\begin{array}{l}\text { Manosque . } \\
\text { Sérignan... }\end{array}$ & $\begin{array}{l}5000 \\
6000\end{array}$ & $\begin{array}{l}28 \\
28\end{array}$ & $\begin{array}{l}6270 \\
3620\end{array}$ & $\begin{array}{l}28 \\
28\end{array}$ & $\begin{array}{l}1,25 \\
0,60\end{array}$ \\
\hline 1973 & $\begin{array}{l}\text { Gréoux ... } \\
\text { Manosque } \\
\text { Sérignan... }\end{array}$ & $\begin{array}{l}4970 \\
1750 \\
5370\end{array}$ & $\begin{array}{l}27 \\
17 \\
27\end{array}$ & $\begin{array}{r}800 \\
2060 \\
2100\end{array}$ & $\begin{array}{l}38 \\
20 \\
23\end{array}$ & $\begin{array}{l}0,22 \\
1,36 \\
0,33\end{array}$ \\
\hline
\end{tabular}

1. Cellules obtenues en été à partir des cellules parent réactivées après hibernation.

2. Rapport $\frac{\text { Nombre de cellules }+ \text { fille. }}{\text { Nombre de cellules } q \text { parent }}$.

1. Zellen, die im Sommer aus den nach der Uberwinterung reaktivierten Elternzellen gewonnen wurden.

2. Verhültnis der Anzahl Tochterzellen (q) zur Anzahl der Elternzellen ( $($ )

de cellules à Sérignan (1973) de la fin du vol de première génération à la fin du vol de deuxième génération. La première génération produit environ 1200 cellules; 740 d'entre elles donnent des individus de deuxième génération qui produisent environ 1140 cellules ce qui représente une multiplication par 1,5 et prouve la grande activité des femelles de deuxième génération en août-septembre. Dans ce cas l'accroissement global d'une génération à l'autre est dans le rapport 1,3 .

Tous ces rapports d'accroissement sont des données à valeur indicative car on voit dans les paragraphes suivants qu'une partie plus ou moins grande de la progéniture est détruite pour diverses raisons. 


\section{III. - Les pertes subies par la progéniture}

\section{A - Accroissement réel de la population}

Les pertes subies par la progéniture sont considérables, puisqu'à l'entrée de l'hibernation les pourcentages de prénymphes saines en diapause s'échelonnent de 6 à $51 \%$ dans les 13 nichoirs expérimentaux (fig. 3). L'accroissement réel de la population (Tableau 8) est par conséquent dans tous les éle-

TABL. 8. - Evolution de la population, compte tenu des pertes subies par la progéniture

Taв. 8. - Populationsentwicklung unter Berücksichtigung der Verluste bei der Nachkommenschaft

\begin{tabular}{|c|c|c|c|c|c|c|}
\hline \multirow{2}{*}{$\begin{array}{l}\text { Année } \\
\text { Jahr }\end{array}$} & \multirow{2}{*}{$\begin{array}{c}\text { Lieu } \\
\text { Ort }\end{array}$} & \multicolumn{2}{|c|}{$\begin{array}{l}\text { Population parent (« éclosoir ») } \\
\text { Elternpopulation ( } \text { Schlüpfräume } ")\end{array}$} & \multicolumn{2}{|c|}{$\begin{array}{l}\text { Population fille }{ }^{1} \text { (nichoirs) } \\
\text { Tochterpopulation (Nistanlagen) }\end{array}$} & $\begin{array}{c}\text { Coefficient } \\
\text { de multiplication } \\
\text { réelle } \\
\text { Koeffizient der } \\
\text { tatsächlichen }\end{array}$ \\
\hline & & $\begin{array}{c}\text { Nombre } \\
\text { de cellules } \\
\text { Zahl d. Zellen }\end{array}$ & $\begin{array}{c}\% \text { de cellules } \\
\text { ㅇ } \\
\% \text { der } q \text { Zellen }\end{array}$ & $\begin{array}{c}\text { Nombre } \\
\text { de cellules } \\
\text { saines en } \\
\text { diapause } \\
\text { Zahl d. unver- } \\
\text { sehrten Zellen } \\
\text { in Diapause }\end{array}$ & $\begin{array}{c}\% \text { de cellules } \\
q \\
\% \text { der } q \text { Zellen }\end{array}$ & ㅇ P.N./q P = \\
\hline 1972 & $\begin{array}{l}\text { Manosque . } \\
\text { Sérignan... }\end{array}$ & $\begin{array}{l}5000 \\
6000\end{array}$ & $\begin{array}{l}28 \\
28\end{array}$ & $\begin{array}{r}1384 \\
790\end{array}$ & $\begin{array}{l}20 \\
12\end{array}$ & $\begin{array}{l}0,20 \\
0,06\end{array}$ \\
\hline 1973 & $\begin{array}{l}\text { Gréoux ... } \\
\text { Manosque . } \\
\text { Sérignan... }\end{array}$ & $\begin{array}{l}4970 \\
1750 \\
5370\end{array}$ & $\begin{array}{l}27 \\
17 \\
27\end{array}$ & $\begin{array}{r}45 \\
1015 \\
1050\end{array}$ & $\begin{array}{l}(38) \\
19 \\
22\end{array}$ & $\begin{array}{l}0,01 \\
0,65 \\
0,16\end{array}$ \\
\hline
\end{tabular}

1. Même légende que pour le tableau 4.

2. Rapport Nombre de prénymphes $ᄋ$ fille saines en diapause

Nombre de cellules $q$ parent.

1. Wie in Tab. 4.

2. Verhältnis der unversehrten Tochter-Pronymphen $(q)$ in Diapause zur Zahl der Elternzellen ( $($ ).

vages, bien inférieur à l'accroissement apparent figuré sur le tableau précédent. Aucun des coefficients de multiplication n'est supérieur à 1 ; aucun élevage ne permet d'accroître la population et le meilleur (Manosque, 1973) -donne un rapport égal à 0,65 .

Les pertes subies par la progéniture sont de trois types :

10 Les ennemis naturels détruisent 10 à $50 \%$ des cellules.

$2^{\circ}$ Pour des raisons indéterminées 3 à $35 \%$ des œufs et des larves meurent.

$3^{\circ}$ Les émergences d'adultes de deuxième (ou de troisième) génération, 

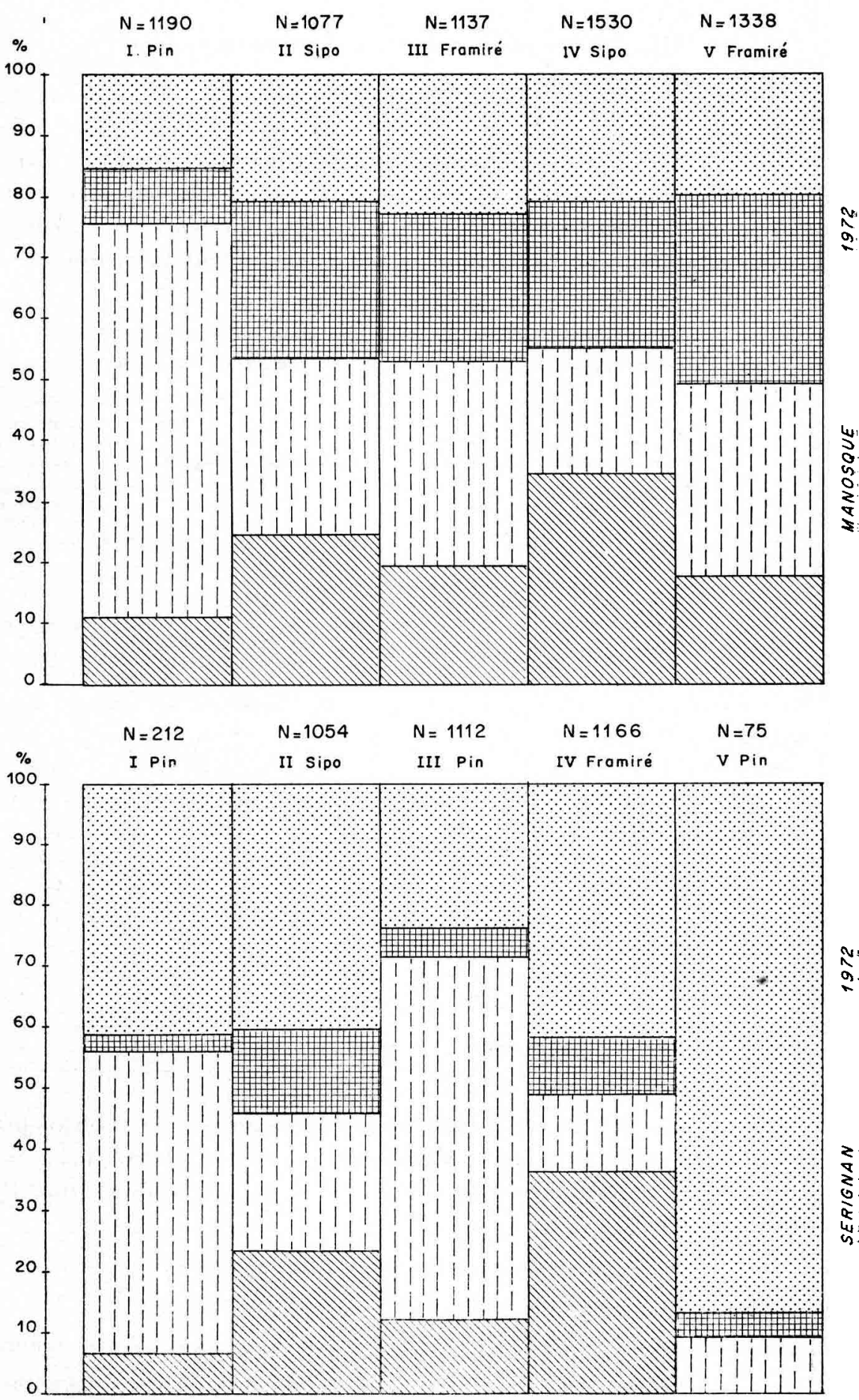


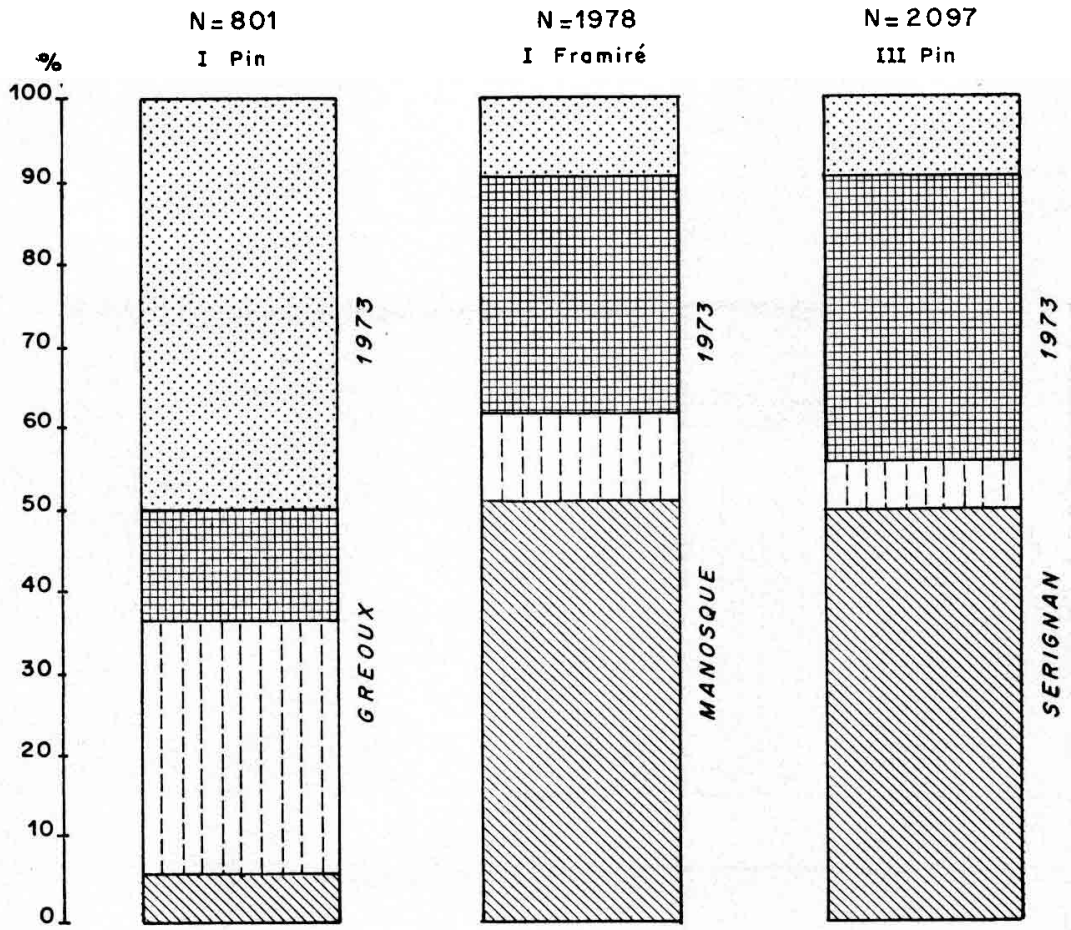

FIg. 3. - Causes des pertes dans la progêniture contenue dans 13 nichoirs de Megachile pacifica Verticalement $=\%$ du total des cellules construites

$\mathbf{N}=$ Nombre total de cellules construites

I à $\mathrm{V}=$ Ordre de rangement de gauche à droite dans l'abri - Bois utilisé

Aвв. 3. - Ursachen der Verluste bei der Nachkommenschaft in 13 Nistanlagen von Megachile pacifica Ordinate $=\%$ der insgesamt gebauten Zellen

$\mathrm{N}=$ Gesamtzahl der gebauten Zellen

I bis $\mathrm{V}=$ Reihenfolge von links nach rechts im Schutzraum - das verwendete Holz

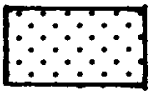

Cellules détruites par les ennemis naturels.

Von natürlichen

Feinden zerstörte Zellen.

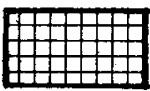

\section{Eufs} et larves morts.

Abgestorbene Eier und Larven.

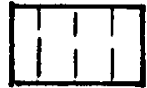

Nymphes, ou adultes émergeant avant hibernation. während der Nymphen oder Diapause. vor der Diapause schlïipfende Adulte.

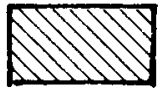

Prénymphes saine en diapause.

Unversehrte

Pronymphen 

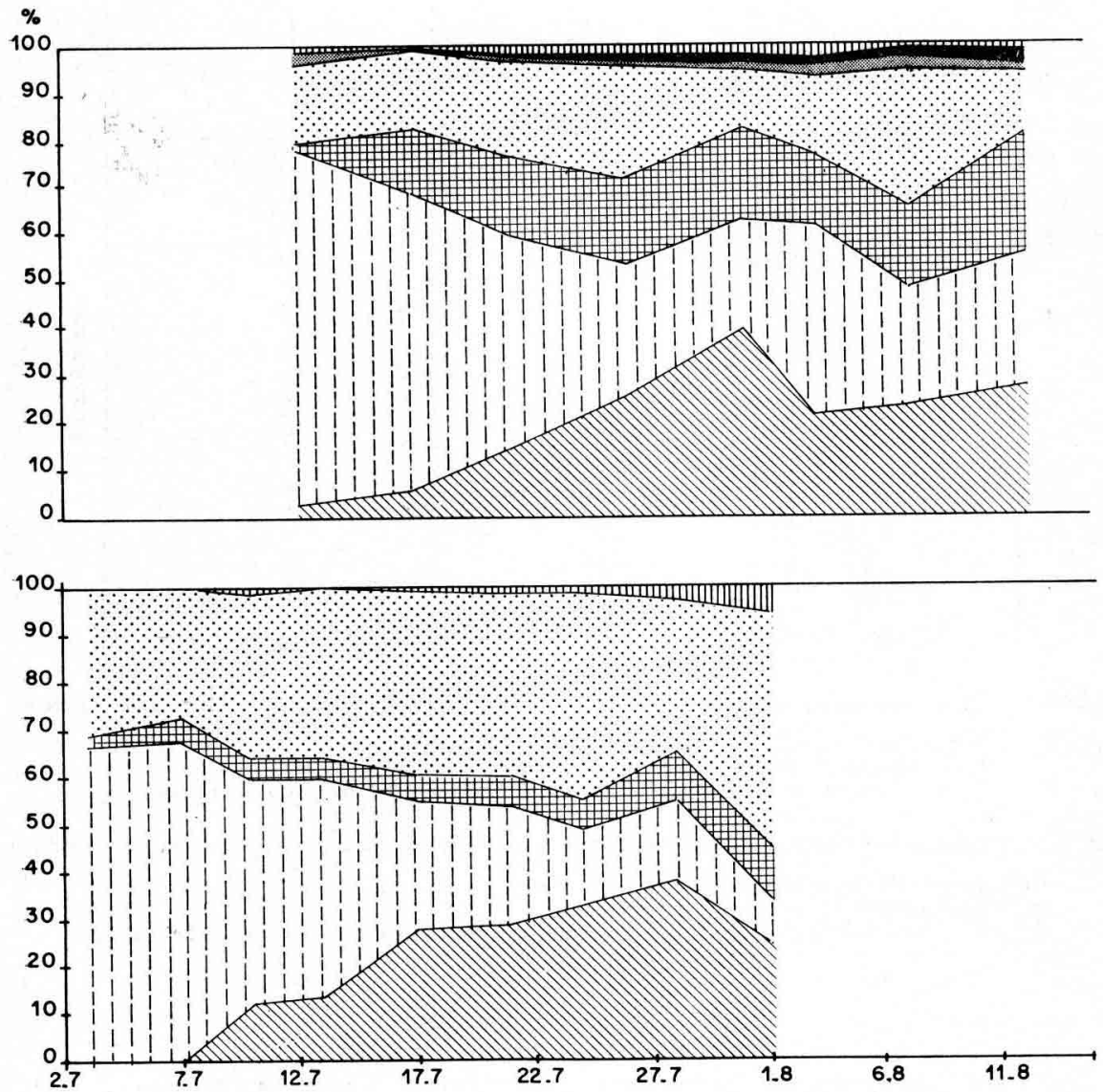

Fig. 4. - Causes de pertes dans la progéniture de Megachile pacifica en fonction de la date de ponte Verticalement \% des cellules construites 

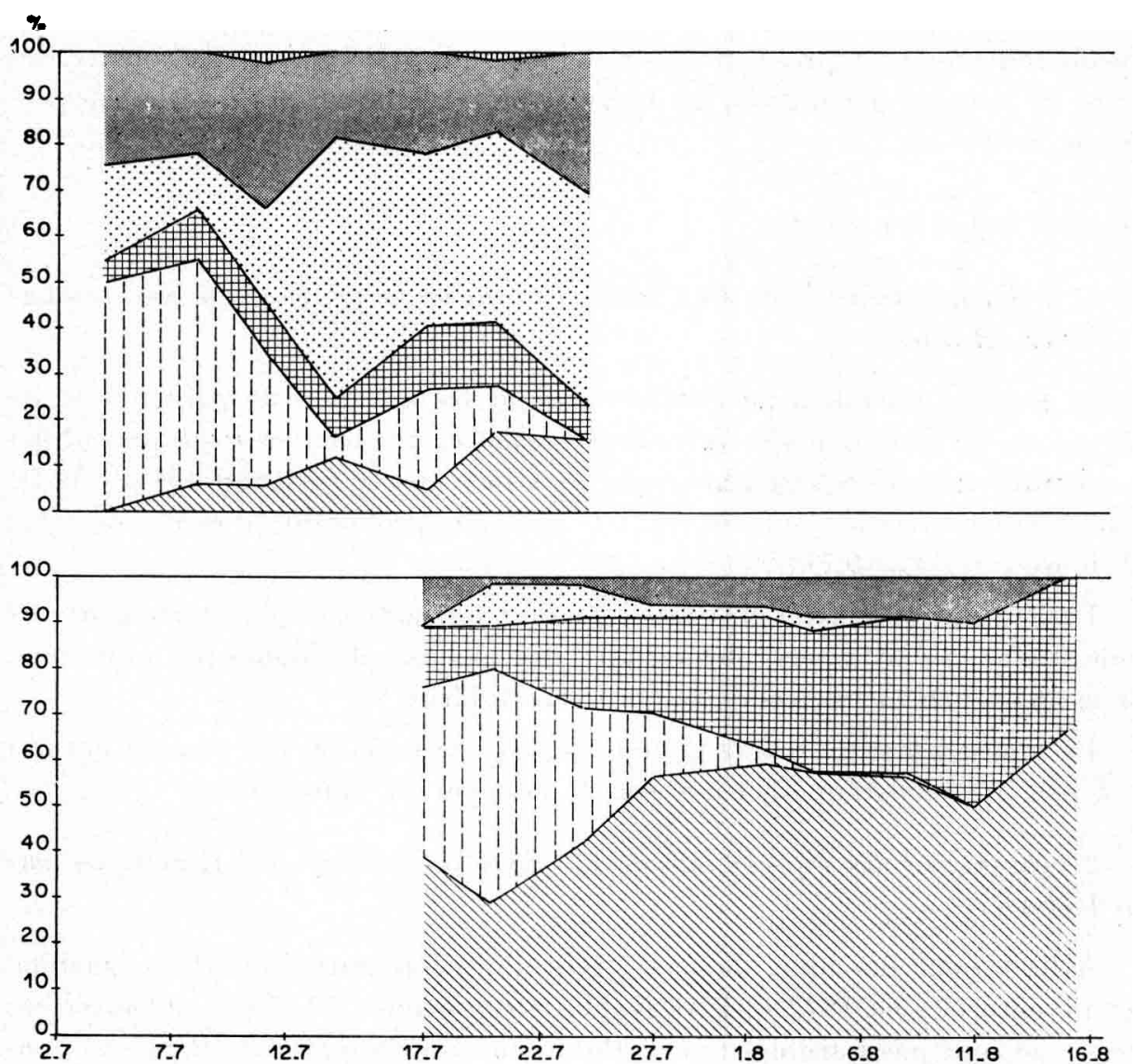

Aвв. 4. - Ursachen der Verluste bei der Nachkommenschaft von Megachile pacifica in Abhängigkeit vom Zeitpunkt der Eiablage

Ordinate $=\%$ der gebauten Zellen

\begin{tabular}{|c|c|c|c|c|c|c|}
\hline \multirow{2}{*}{$\begin{array}{r}\text { Prénymphes } \\
\text { saines } \\
\text { en diapause }\end{array}$} & \multirow{2}{*}{$\begin{array}{r}\text { Nymphes } \\
\text { ou adultes } \\
\text { émergeant } \\
\text { avant } \\
\text { hibernation }\end{array}$} & \multirow{2}{*}{$\begin{array}{r}\text { CEufs } \\
\text { et larves } \\
\text { morts }\end{array}$} & \multicolumn{3}{|c|}{$\begin{array}{c}\text { Cellules } \\
\text { détruites } \\
\text { par }\end{array}$} & \multirow[t]{2}{*}{$\begin{array}{r}\text { Cellules } \\
\text { détruites } \\
\text { par } \\
\text { ennemis } \\
\text { naturels } \\
\text { secondaires }\end{array}$} \\
\hline & & & Trichodes & Miltogramma & Melittobia & \\
\hline EDOZ & $E=-$ & 䎴 & & & & ए马 \\
\hline \multirow[t]{2}{*}{$\begin{array}{l}\text { Unversehrte } \\
\text { Pronymphen } \\
\text { in Diapause }\end{array}$} & \multirow{2}{*}{$\begin{array}{l}\text { Nymphen oder } \\
\text { vor der } \\
\text { Uberwinterung } \\
\text { geschlüpfte } \\
\text { Adulte }\end{array}$} & \multirow[t]{2}{*}{$\begin{array}{l}\text { Abgestorbene } \\
\text { Eier und } \\
\text { Larven }\end{array}$} & & $\begin{array}{c}\text { Zellen } \\
\text { zerstört } \\
\text { durch }\end{array}$ & & \multirow[t]{2}{*}{$\begin{array}{l}\text { Durch } \\
\text { Feinde } \\
\text { zerstörte } \\
\text { Zellen }\end{array}$} \\
\hline & & & Trichodes & Miltogramma & Mellitobia & \\
\hline
\end{tabular}


survenant pendant la période de stockage précédant l'hibernation, amoindrissent la population de 6 à $64 \%$.

La figure 4 met en évidence la liaison existant entre la date de ponte et la probabilité pour la progéniture de donner une prénymphe en diapause, un adulte de seconde génération, un individu non viable ou parasité, ou dévoré par un prédateur.

\section{B - Les ennemis naturels}

1. Influence du bois, du lieu et du moment de la ponte sur le pourcentage de cellules détruites.

Le bois du nichoir a un effet certain sur les attaques de parasites et de prédateurs. Le Pin est moins favorable à celles-ci que les bois exotiques : Sipo et Framiré (tableau 9). Ce phénomène est bien net à Sérignan $\left(\chi^{2}=24,2\right)$, il est atténué, mais toujours significatif avec une probabilité d'erreur de $1 \%$, à Manosque $\left(\chi^{2}=9,7\right)$.

Le lieu où est placé l'abri est le facteur qui influe le plus fortement sur l'importance des attaques : les cellules des nichoirs de Manosque sont deux fois moins touchées que celles de Sérignan (tableau 9).

La figure 4 montre que le pourcentage de destruction par ennemi naturel est à peu près constant quel que soit le moment de nidification.

2. Inventaire des espèces rencontrées dans les nichoirs - Remarques sur leur biologie.

A l'intérieur des nids, douze espèces d'insectes autres que le mégachile, sont inventoriées en 1972 et 1973 (tableau 10) (photos Pl. 4 et 5). Toutes ces espèces ne sont pas nuisibles : ainsi Ptinus fur L (Ptinidae) et Attagenus pantherinus Ahr. (Dermestidae) ne semblent pas s'attaquer à la progéniture tant qu'elle est vivante. Par ailleurs les espèces réellement nuisibles ne sont pas toutes aussi actives les unes que les autres : les prédateurs tels que les larves de Trichodes font individuellement beaucoup plus de tort à la population d'abeilles que les parasites tels que les larves de Sapyga ou de Stelis. C'est pour cette raison que l'importance de chaque espèce est exprimée en pourcentage de cellules détruites et non en pourcentage d'individus.

Le tableau 10 fait apparaître la constance et l'importance des attaques de Trichodes apiarus L. (40,1 à 95, $1 \%$ des cellules détruites). Le diptère Miltogramma murinum Mg. (Tachinidae) $(0,4$ à 58,6 \%) est moins constant mais les effets de son parasitisme ne sont pas négligeables. Melittobia acasta Walk (Eurytomidae) ne parasite que 0,6 à 13,5\% des cellules. Sapyga quinquepunctata F. (Sapygidae), Stelis phaeoptera Kirb, Coelioxys elongata Lep. (Megachilidae), Monodontomerus obscurus Westw. (Torymidae) et Ptero- 
TABL. 9. - Destruction des cellules par les ennemis naturels en fonction du lieu et du bois des nichoirs en 1972

TABL. 9. - Zerstörung der Zellen durch natïliche Feinde in Abhängigkeit von Ort und Holzart der Nistanlage (1972)

\begin{tabular}{|c|c|c|c|c|}
\hline \multirow[b]{2}{*}{$\begin{array}{l}\text { Bois } \\
\text { Holzart }\end{array}$} & \multicolumn{2}{|c|}{ Manosque } & \multicolumn{2}{|c|}{ Sérignan } \\
\hline & $\%^{1}$ & $N^{2}$ & $\%$ & $\mathbf{N}$ \\
\hline Pin & 15,5 & 1190 & 29,6 & 1399 \\
\hline Sipo & 20,7 & 2607 & 40,1 & 1054 \\
\hline Framiré $\ldots \ldots \ldots$ & 27,7 & 24.75 & 41,6 & 1166 \\
\hline
\end{tabular}

1. Pourcentage de cellules détruites.

2. Nombre total de cellules construites.

1. \% der vernichteten Zellen.

2. Gesamtzahl der gebauten Zellen.

TABL. 10. - Pertes causées par les ennemis naturels

TAB. 10. - Durch die natïrlichen Feinde verursachte Verluste

\begin{tabular}{|c|c|c|c|c|c|c|c|c|c|c|c|c|c|c|}
\hline & & \multicolumn{12}{|c|}{$\begin{array}{l}\% \text { des cellules détruites } \\
\% \text { der zerstörten Zellen }\end{array}$} & \multirow{3}{*}{$\begin{array}{c}\text { Total } \\
\text { des cellules } \\
\text { détruites } \\
\text { Gesamatzahl } \\
\text { d. zerstörten } \\
\text { Zellen }\end{array}$} \\
\hline & & \multicolumn{4}{|c|}{ Coleoptera } & \multicolumn{2}{|c|}{ Diptera } & \multicolumn{5}{|c|}{ Hymenoptera } & $\begin{array}{l}\text { Lepi- } \\
\text { doptera }\end{array}$ & \\
\hline $\begin{array}{l}\text { Année } \\
\text { Jahr }\end{array}$ & $\underset{\text { Art }}{\text { Espèce }}$ & 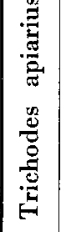 & 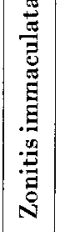 & 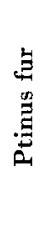 & 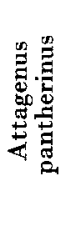 & 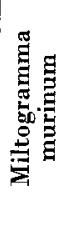 & 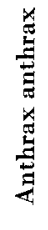 & 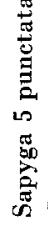 & 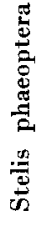 & 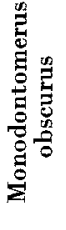 & 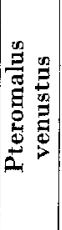 & 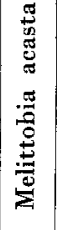 & $\mathrm{X}$ & \\
\hline 1972 & $\begin{array}{l}\text { Manosque. } \\
\text { Sérignan .. }\end{array}$ & $\begin{array}{l}74,2 \\
95,1\end{array}$ & - & $\begin{array}{l}0,2 \\
-\end{array}$ & $\begin{array}{l}1,3 \\
-\end{array}$ & $\begin{array}{l}6,6 \\
0,4\end{array}$ & - & $\begin{array}{l}1,8 \\
0,1\end{array}$ & $\overline{1,9}$ & $\begin{array}{l}2,8 \\
0,1\end{array}$ & $\begin{array}{l}1,7 \\
--\end{array}$ & $\begin{array}{r}11,0 \\
2,1\end{array}$ & $\begin{array}{l}0,4 \\
0,3\end{array}$ & $\begin{array}{l}1250 \\
1322\end{array}$ \\
\hline 1973 & $\begin{array}{l}\text { Gréoux ... } \\
\text { Manosque } \\
\text { Sérignan . }\end{array}$ & $\left|\begin{array}{l}52,3 \\
40,1 \\
55,4\end{array}\right|$ & $\begin{array}{l}1,0 \\
- \\
-\end{array}$ & $\begin{array}{l}- \\
- \\
-\end{array}$ & - & $\begin{array}{l}46,1 \\
58,6 \\
19,7\end{array}$ & $\begin{array}{l}0,3 \\
- \\
0,5\end{array}$ & $\begin{array}{l}- \\
- \\
2,1\end{array}$ & $\begin{array}{c}- \\
\overline{7,3}\end{array}$ & $\begin{array}{l}- \\
- \\
-\end{array}$ & $-\frac{-}{1,6}$ & $\begin{array}{r}0,3 \\
1,4 \\
13,4\end{array}$ & - & $\begin{array}{l}397 \\
222 \\
192\end{array}$ \\
\hline
\end{tabular}




\section{PLANCHE IV}

Parasites et Prédateurs de M. pacifica (Photos S. CARRÉ)

a) 1. Physocephala pusilla

(diptère Conopidae) b) Anthrax anthrax (diptère Bombyliidae)

2. femelle de $M$. pacifica parasitée par P. pusilla

c) Miltogramma murinum

(diptère Tachinidae et sa pupe)

e) Trichodes apiarius

(larve)

d) Trichodes apiarius

(Coléoptère Cleridae)

f) Zonitis immaculata

(Coléoptère Meloidae)

Trait blanc

ou noir $-4 \mathrm{~mm}$

TAFEL IV

Parasiten und Räuber von Megachile pacifica (phot. S. CARRÉ)

a) 1. Physocephala pusilla

(Diptera, Conopidae)

b) Anthrax anthrax

(Diptera, Bombylidae)

2. $q$ von M. pacifica

befallen von $P h$. pusilla

c) Miltogramma murinum

d) Trichodes apiarius

(Diptera, Tachinidae mit Puppe)

(Coleoptera, Meloidae)

e) Trichodes apiarius

f) Zonitis immaculata

Larve

(Coleoptera, Meloidae)

weisser oder schwarzer Strich $-4 \mathrm{~mm}$ 

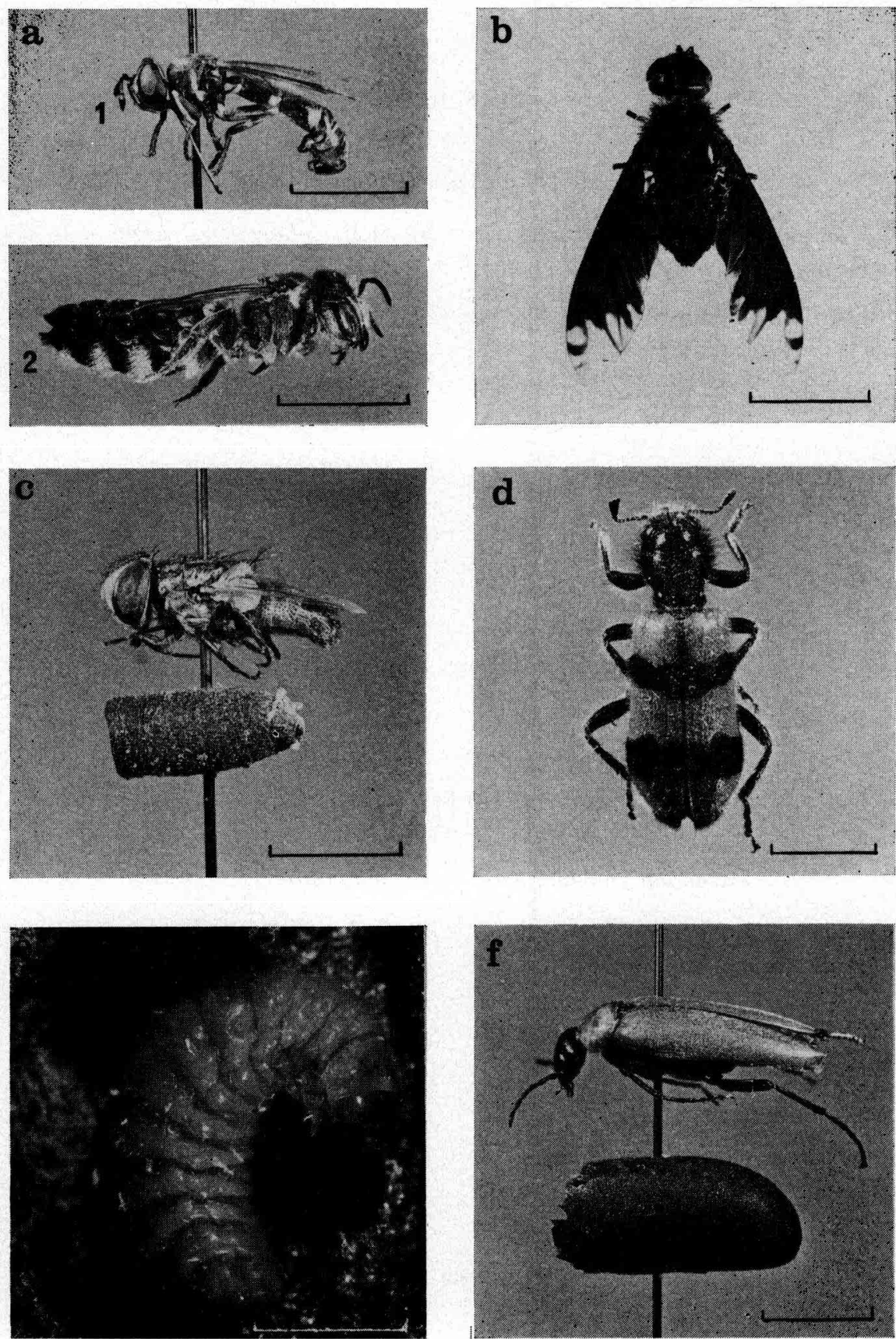


\section{PLANCHE V}

Parasites de M. pacifica (Photos S. Carré)

a) Coelioxys elongata mâle

(Hym. Megachilidae)

c) Sapyga 5 punctata avec son cocon

(Hym. Sapygidae)

$($ Hym. Torymidae $)$
1. Femelle
2. Mâle
$f)$ Mellittobia acasta
$($ Hym. Eulophidae $)$
1. Femelle
2. Mâle
Trait blanc $\longmapsto=$
ou noir

b) Stelis phaeoptera avec son cocon (Hym. Megachilidae)

d) Monodontomerus obscurus

(Hym. Torymidae)

1. Femelle

2. Mâle

e) Pteromalus venustus

(Hym. Pteromalidae)
Mellittobia acasta

1. Femelle

2. Mâle

ou noir

\section{TAFEL V}

Parasiten von Megachile pacifica (phot. S. Carré)

a) Coelioxys elongata ${ }^{\star}$

(Hym. Megachilidae)

c) Sapyga quinquepunctata

(Hym. Sapygidae)

mit Kokon

e) Pteromalus venustus

(Hym. Pteromalidae) b) Stelis phaeoptera

(Hym. Megachilidae) mit Kokon

d) Monodontomerus obscurus (Hym. Torymidae)

1 - $92-\hat{\jmath}$

f) Melittobia acasta

(Hym. Eulophidae)

1 - 2 -ð

schwarzer oder weisser Strich $\downarrow-!=4 \mathrm{~mm}$ 

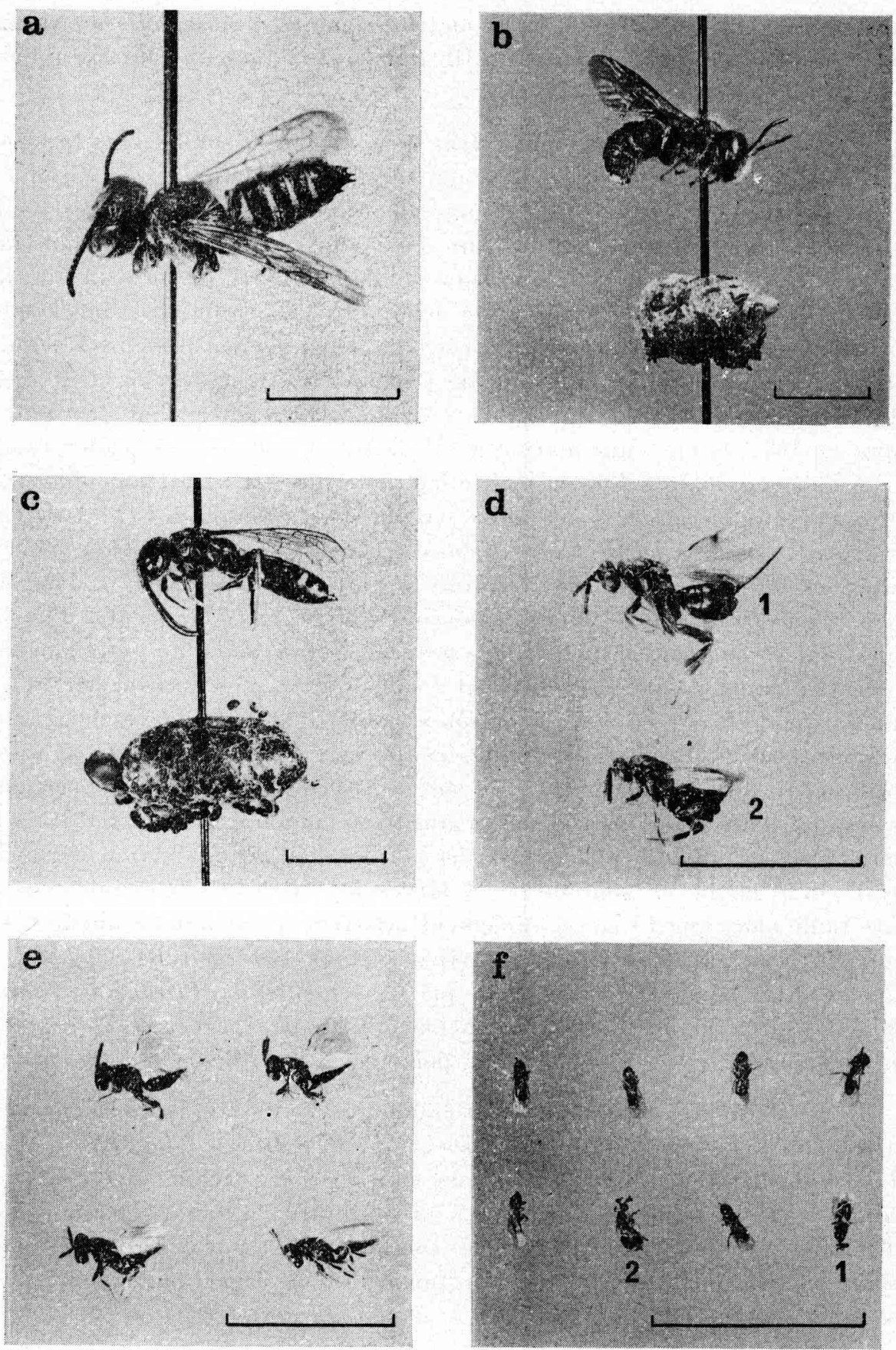
malus venustus Wlk (Pteromalidae) sont des espèces secondaires ne causant jamais plus de 7,3\% de dégâts. Enfin on peut signaler Anthrax anthrax Shrank (Bombyliidae) et Zonitis immaculata Ol. (Meloïdae) dont un seul exemplaire est issu de l'ensemble des nichoirs.

a) Trichodes apiarius se trouve dans les nids de mégachiles sous la forme larvaire. Les individus de couleur rouge corail sont de taille très variable : 3 à $30 \mathrm{~mm}$. Ils sont à la fois prédateurs et pollinivores. Deux mois après l'infestation on voit les larves soit en train de se nourrir, soit au repos dans une loge qu'elles confectionnent en amalgamant des rognures de bois du nichoir. Au cours de 2 années d'observation on dénombre 2588 cellules de mégachiles détruites par 654 larves de $T$. apiarius, soit en moyenne près de 4 cellules détruites par une larve. L'intensité de l'attaque dépend de l'ensoleillement de la surface des nichoirs (tableau 11). Lorsque le haut des nichoirs est ombré comme en 1972, la moyenne d'attaque est de $16,3 \%$, dans cette partie, tandis qu'elle s'élève à $26,3 \%$ dans la moitié inférieure qui est nettement plus ensoleillée. Lorsque les auvents des abris sont enlevés comme en 1973, l'attaque est identique dans la partie basse et dans la partie haute $: 14,7 \%$ et $14,5 \%$. Toutes les larves de Trichodes récoltées à l'automne sont élevées dans des boîtes de polystyrène de 3 à $4 \mathbf{c m}^{3}$. Elles sont nourries avec des pelotes d'abeille domestique et peuvent bâtir leur loge grâce à la présence d'un petit morceau de contre-plaqué. Les larves hibernent 5 mois à $5{ }^{\circ} \mathrm{C}$ puis sont réactivées à la température de $19{ }^{\circ} \mathrm{C}$. Au bout d'environ 45 jours, $29,2 \%$ des larves deviennent des adultes. Tous les autres individus continuent à se nourrir sans se métamorphoser jusqu'à l'hibernation suivante. Au cours de l'élevage certaines larves noircissent et meurent. Aucun parasitisme ne paraît les affecter. Dans les 2 petits pièges à appât de pollen, installés sous les nichoirs de l'abri de Sérignan, (1973), on recueille en septembre 34 larves de Trichodes, généralement de petite taille alors que 19 larves seulement infestent les nichoirs à abeilles. Les larves sont “ piégées " au fond des cavités, surtout dans les tubes de $7 \mathrm{~mm}$ de diamètre. Ceci démontre d'une part que les femelles de Trichodes viennent pondre à l'intérieur des nids de mégachiles et d'autre part qu'elles sont fortement attirées par les provisions de pollen.

b) Miltogramma murinum est un endoparasite des larves de $M$. pacifica. $\mathrm{Il}$ arrive que l'on trouve plusieurs pupes brunes de $8 \mathrm{~mm}$ de long dans la même cellule d'abeille; cependant en moyenne une larve de tachinaire détruit une larve de mégachile puisque l'on trouve au cours des 2 années 474 pupes dans 440 cellules. Certaines pupes sont vides comme si des adultes de diptère émergeaient en deuxième génération. On remarque un léger parasitisme du à Melittobia acasta.

c) Anthrax (= Argyromoeba) Anthrax est ectoparasite des larves. En deux ans on a trouvé 2 individus dans 2 cellules. 
TABL. 11. -- Dégâts causés par Trichodes apiarius en fonction de l'ensoleillement des nichoirs TAB. 11. - Durch Trichodes apiarius verursachte Schäden in Abhängigkeit von der Sonnenbestrahlung der Nistanlagen

\begin{tabular}{|c|c|c|c|c|c|c|c|c|c|c|c|}
\hline & $\begin{array}{c}\text { Année } \\
\text { Jahr }\end{array}$ & \multicolumn{10}{|c|}{1972} \\
\hline & $\begin{array}{c}\text { Lieu } \\
\text { Ort }\end{array}$ & \multicolumn{5}{|c|}{ Manosque } & \multicolumn{4}{|c|}{ Sérignan } & \\
\hline \multicolumn{2}{|c|}{$\begin{array}{l}\text { Nombre total de nids } \\
\text { construits } \ldots \ldots \ldots \ldots \\
\text { Gesamtzahl d. gebanten } \\
\text { Nester }\end{array}$} & \multicolumn{5}{|c|}{1.588} & \multicolumn{4}{|c|}{822} & \\
\hline \multicolumn{2}{|c|}{$\begin{array}{l}\text { Bois du nichoir } \\
\text { Holz d. Nistanlage }\end{array}$} & Pin & Sipo & Framiré & Sipo & Framiré & Pin & Sipo & Pin & Framiré & Moyenne \\
\hline 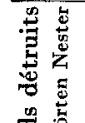 & $\begin{array}{l}\text { Moitié }{ }^{1} \quad \text { supé- } \\
\text { rieure } \quad \ldots \ldots \ldots \ldots \\
\text { obere Hälfte }\end{array}$ & 9,3 & 12,4 & 12,5 & 4,4 & 8,6 & 14,3 & 34,4 & 21,4 & 38,0 & 16,3 \\
\hline 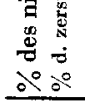 & $\begin{array}{l}\text { Moitié inférieu- } \\
\text { re .......... } \\
\text { untere Hälfte }\end{array}$ & 13,4 & 23,5 & 22,0 & 20,2 & 18,8 & 46,9 & 40,0 & 26,3 & 42,2 & 26,3 \\
\hline & $\begin{array}{l}\text { Année } \\
\text { Jahr }\end{array}$ & & & & & & 19 & & & & \\
\hline & $\begin{array}{c}\text { Lieu } \\
\text { Ort }\end{array}$ & & & Gréoux & & Manosqu & & & rignan & & \\
\hline $\begin{array}{l}\text { Nombr } \\
\text { Gesamtz }\end{array}$ & $\begin{array}{l}\text { total de nids cor } \\
\text { ahl d. gebauten Nest }\end{array}$ & istruit & & 179 & & 386 & & & 463 & & \\
\hline $\begin{array}{l}\text { Bois du } \\
\text { Holz d. }\end{array}$ & $\begin{array}{l}\underset{\text { nichoir }}{\text { Nistanlage }} \\
\text { ista }\end{array}$ & & & Pin & & Framire & & & Pin & & Moyenne \\
\hline 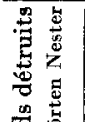 & $\begin{array}{l}\text { Moitié }{ }^{1} \text { supérieu } \\
\text { obere Hälfte }\end{array}$ & re. & & 25,0 & & 4,0 & & & 3,0 & & 14,7 \\
\hline  & $\begin{array}{l}\text { Moitié inférieure } \\
\text { untere Hälfte }\end{array}$ & & & 26,8 & & 2,6 & & & 6,0 & & 14,5 \\
\hline
\end{tabular}

1. La moitié supérieure est moins ensoleillée que la moitié inférieure dans les nichoirs de 1972 placés dans des abris à auvent.

1. 1972 ist die obere Hälfte der Nistanlagen der Sonnenbestrahlung weniger ausgesetzt gewesen, weil sie durch ein Dach abgesehirmt waren. 
d) Melittobia acasta est un microchalcidien ectoparasite des larves et des nymphes. On trouve de 30 à 125 larves de Melittobia sur chaque abeille. La rapidité de développement de ce parasite ne paraît pas provoquer les ravages que l'on peut craindre.

e) Pteromalus venustus est aussi un chalcidien ectoparasite, de plus grande taille que Melittobia. Les larves de mégachiles parasitées fournissent assez de nourriture pour alimenter 18 larves de Pteromalus en moyenne. L'inspection de 12 cellules montre que le nombre de larves de parasites varie de 8 à 31 . Par ailleurs dans certaines cellules on rencontre en mélange des Pteromalus et des Monodontomerus ou bien des Pteromalus et des Melittobia.

f) Monodontomerus obscurus est le plus gros des chalcidiens ectoparasites de $M$. pacifica. On trouve en moyenne 5 larves par cellule. Dans 11 cellules examinées on dénombre de 2 à 15 larves. Une deuxième génération partielle apparaît un mois après la ponte.

g) Sapyga 5 punctata est un cleptoparasite à excréments noirs caractéristiques. La larve dévore la provision de pollen destinée à l'abeille, puis tisse un cocon allongé et brillant. A la fin de l'été le cocon contient un adulte en diapause qui doit hiberner puis être réactivé avant d'émerger.

h) Stelis phaeoptera est aussi un cleptoparasite. Son cocon est facilement reconnaissable grâce à son feutrage externe blanc et son petit cône antérieur. Cette espèce hiberne à l'état de prénymphe en diapause.

i) Coelioxys elongata est le troisième cleptoparasite. L'allure générale de l'adulte rappelle celle des mégachiles. Le cocon tissé par la larve ressemble aussi beaucoup à un cocon de $M$. pacifica et passe inaperçu lors du tri général. Cette espèce rencontrée seulement en 1972 à Manosque a deux générations par an. La deuxième génération partielle émerge en même temps que la deuxième génération de $M$. pacifica.

j) Zonitis immaculata est un melö̈de pollinivore hibernant à l'état de larve en diapause.

Après l'hibernation les délais d'émergence de ces ennemis naturels sont très variables d'une espèce à l'autre (tableau 12). Certaines espèces sont plus précoces que $M$. pacifica comme les chalcidiens (Melittobia, Pteromalus, Monodontomerus) d'autres sont plus tardives (Trichodes, Stelis).

L'importance des dégâts occasionnés par les ennemis naturels dépend surtout du lieu et de l'année, secondairement du bois. Le Pin semble moins attractif que les bois exotiques. Parmi les 12 espèces rencontrées dans les nids, seul le prédateur Trichodes apiarius cause constamment des destructions sérieuses. L'emploi d'appâts à base de pollen en pelote permet de sauvegarder 
dans une certaine mesure les nids de mégachiles. La tachinaire Miltogramma murinum peut causer des dégâts non négligeables. Les attaques de cette espèce sont assez inconstantes.

TABL. 12. - Délais d'émergence à $24{ }^{\circ} \mathrm{C}$ de quelques ennemis naturels ayant hiberné 3 mois à $5{ }^{\circ} \mathrm{C}$ TAB. 12. - Verzögerung des Schlüpfens einiger natürlicher Feinde bei $24{ }^{\circ} \mathrm{C}$ nach dreimonatiger Uberwinterung bei $5{ }^{\circ} \mathrm{C}$

\begin{tabular}{|c|c|c|}
\hline Melittobia acasta $\ldots \ldots \ldots \ldots \ldots \ldots \ldots \ldots \ldots \ldots$ & \multicolumn{2}{|c|}{6 à 13 jours (Tage) } \\
\hline Pteromalus venustus $\ldots \ldots \ldots \ldots \ldots \ldots \ldots \ldots$ & 10 à 13 & « \\
\hline Monodontomerus obscurus $\ldots \ldots \ldots \ldots \ldots \ldots \ldots \ldots$ & 13 à 20 & $\ll$ \\
\hline 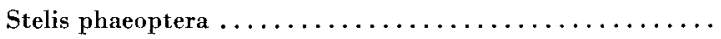 & 35 à 45 & $\ll$ \\
\hline Sapyga quinquepunctata ........... & 13 à 23 & 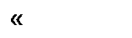 \\
\hline Miltogramma murinum $\ldots \ldots \ldots \ldots \ldots \ldots \ldots \ldots$ & 35 à 41 & $\ll$ \\
\hline Physocephala pusilla $\ldots \ldots \ldots \ldots \ldots \ldots$ & 30 & $\ll$ \\
\hline 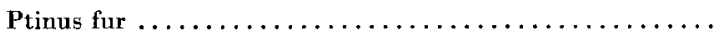 & 30 & « \\
\hline 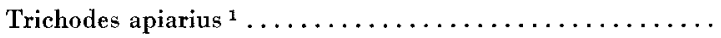 & 50 & $\ll$ \\
\hline
\end{tabular}

1. Il s'agit des individus qui effectuent leur mue imaginale après un seul hiver.

1. Es handelt sich um Tiere, die nach nur einem Winter ihre Imaginalhäutung durchmachen.

\section{C - La mortalité chez les oufs et les larves}

A l'ouverture des cellules on trouve parfois les restes du chorion d'un cuf ou bien ceux d'une jeune larve, avec les restes de la provision de pollen. Chez d'autres cellules la prénymphe est morte à l'intérieur du cocon. L'analyse globale de la mortalité chez ces stades immatures conduit à plusieurs conclusions :

$1^{0}$ Le Pin favorise la survie des larves de megachile quelque soit le lieu et l'année (4,8 à 9,9\% de mortalité) tandis que le Sipo et le Framiré sont défavorables à la survie $(9,4$ à $28,1 \%$ de mortalité) (tableau 13). La différence de répartition de la mortalité suivant le bois testée par un $\chi^{2}$ est très fortement significative : par exemple si l'on considère tous les nichoirs de 1972 le $\chi^{2}=348$; si l'on considère seulement les nichoirs d'Avignon en 1972 le $\chi^{2}=31$ (d.d.l. =2).

$2^{\circ}$ En 1972 la mortalité à Manosque est environ deux fois plus élevée qu’à Sérignan, quelque soit le bois du nichoir (tableau 13). Cette différence est peut-être liée à la différence des températures nocturnes, plus basses à Manosque (tableau 14).

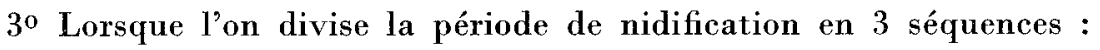

I : Début de la nidification

II : Nidification intense

III : Fin de la nidification

on constate que les chances de survie des oufs et des larves décroissent au fur et à mesure que l'on se rapproche du terme de la ponte (fig. 4 et tableau 14). 
TABL. 13. - Mortalité des aufs et des larves de la première gênération, en fonction du lieu et du bois

TAB. 13. - Sterblichkeit der Eier und Larven der 1. Generation in Abhängigkeit von Ort und Holzart

\begin{tabular}{|c|c|c|c|c|c|c|c|}
\hline \multirow{2}{*}{ Bois } & \multicolumn{2}{|l|}{ Pin } & \multicolumn{2}{|c|}{ Sipo } & \multicolumn{2}{|c|}{ Framiré } & \multirow{2}{*}{$\begin{array}{c}\begin{array}{c}\text { Moyenne } \\
\text { Durchschnitt }\end{array} \\
\% \text { Oe }+\mathrm{L}\end{array}$} \\
\hline & $\% \mathrm{Oe}+\mathrm{L}^{1}$ & $\mathrm{~N}^{2}$ & $\% \mathrm{Oe}+\mathbf{L}$ & $\mathrm{N}$ & $\% \mathbf{O e}+\mathbf{L}$ & $\mathrm{N}$ & \\
\hline Sérignan 1972 & 4,8 & 1399 & 13,9 & 1054 & 9,4 & 1166 & 8,9 \\
\hline Manosque $1972 \ldots$ & 8,9 & 1190 & 24,9 & 2607 & 27,7 & 2475 & 23,0 \\
\hline Manosque 1973 & 5,4 & 74 & - & - & 28,1 & 1984 & 27,3 \\
\hline Gréoux $1973 \ldots \ldots$ & 13,4 & 801 & - & - & - & - & 13,4 \\
\hline \multicolumn{8}{|c|}{$\begin{array}{l}\text { 1. Pourcentage d'œufs et de larves morts. } \\
\text { 2. Nombre total de cellules construites. }\end{array}$} \\
\hline $\begin{array}{l}\text { 1. \% abgestorbener } \\
\text { 2. Gesamatzahl der g }\end{array}$ & und Larven. & & & & & & \\
\hline
\end{tabular}

L'hétérogénéité de la répartition de la mortalité au cours des trois phases de la nidification à Manosque (1972) et Manosque (1973) est nettement significative avec une probabilité de $99 \%$. Les $\chi^{2}$ sont respectivement égaux à 14,4 et 23,5. Dans le cas de Sérignan 1972 le $\chi^{2}$ est égal à 5,7 , c'est-à-dire très proche du seuil de signification à $95 \%$ de probabilité.

4. Si l'on compare les mortalités moyennes figurant dans les tableaux 13 et 14 on remarque que les pertes sont moins élevées lorsque l'on ne tient compte que des nids operculés. Lorsque l'on considère la totalité des nids (operculés et non operculés) la mortalité est plus forte, cela veut dire que les nids non operculés subissent beaucoup plus de pertes que les nids operculés.

La survie des cufs et des larves de première génération est donc favorisée par l'emploi de nichoirs en Pin. Elle est meilleure dans les nids operculés. Elle décroît progressivement du début à la fin de la période de ponte.

\section{$\mathrm{D}$ - Les émergences donnant la deuxième (ou troisième) génération partielle.}

Pendant la phase de stockage des nids d'environ 1 mois, à $20-23{ }^{\circ} \mathrm{C}$ qui précède l'hibernation, le développement larvaire s'achève et en fin de période certains individus deviennent adulte et sortent des cellules. Ils ne peuvent ni hiberner, ni se nourrir et fonder de nids et meurent. Le reste des cellules saines est constitué par les prénymphes en diapause qui doivent hiberner avant de poursuivre leur développement. 
TaBL. 14. - Mortalité chez les aufs et les larves dans les nids oporculés, en fonction de la date de ponte et des températures extrêmes

TAB. 14. - Sterblichkeit der Eier und Larven in den verdeckelten Nestern abhängig vom Zeitpunkt der Eiablage und den Höchst-u. Tiefsttemperaturen

\begin{tabular}{|c|c|c|c|c|c|c|}
\hline 离 & 氶 & $\begin{array}{l}\text { Période } \\
\text { Periode }\end{array}$ & $\begin{array}{c}\text { I } \\
\text { Début } \\
\text { de nidification } \\
\text { Beginn } \\
\text { d. Nestbaues }\end{array}$ & $\begin{array}{c}\text { II } \\
\text { Nidification } \\
\text { intense } \\
\text { Lebhafter } \\
\text { Nestbau }\end{array}$ & $\begin{array}{c}\text { III } \\
\text { Fin de } \\
\text { la nidification } \\
\text { Ende } \\
\text { d. Nestbaues }\end{array}$ & $\begin{array}{c}\text { Moyenne } \\
\text { Durchsehnitt }\end{array}$ \\
\hline \multirow{6}{*}{$\underset{\sim}{\stackrel{\Im}{\Xi}}$} & \multirow{3}{*}{ 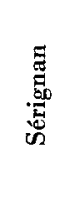 } & $\begin{array}{c}\text { Dates } \\
\theta \mathbf{M}-\theta \mathbf{m}^{1}\end{array}$ & $\begin{array}{c}3-7 / 10-7 \\
32-13\end{array}$ & $\begin{array}{c}11-7 / 21-7 \\
33-14\end{array}$ & $\begin{array}{c}22-7 / 1-8 \\
32-17\end{array}$ & \\
\hline & & $\mathrm{N}^{2}$ & 704 & 1196 & 576 & \\
\hline & & $\% \mathrm{Oe}+\mathrm{L}^{3}$ & 4,0 & 6,0 & 8,5 & 6,0 \\
\hline & \multirow{3}{*}{ 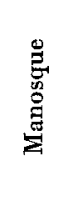 } & $\begin{array}{c}\text { Dates } \\
0 \mathbf{M}-\theta \mathrm{m}\end{array}$ & $\begin{array}{c}12-7 / 21-7 \\
33-8\end{array}$ & $\begin{array}{c}22-7 / 31-7 \\
32-7\end{array}$ & $\begin{array}{c}1-8 / 12-8 \\
34-6\end{array}$ & \\
\hline & & $\mathbf{N}$ & 1687 & 1788 & 1450 & \\
\hline & & $\% \mathrm{Oe}+\mathrm{L}$ & 14,5 & 18,6 & 21,9 & 18,1 \\
\hline \multirow{3}{*}{$\stackrel{\Re}{5}$} & \multirow{3}{*}{ 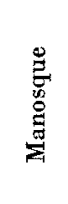 } & $\begin{array}{l}\text { Dates } \\
0 \mathrm{M}-\vartheta \mathrm{m}\end{array}$ & $\begin{array}{c}14-7 / 25-7 \\
30-8\end{array}$ & $\begin{array}{c}26-7 / 2-8 \\
34-6\end{array}$ & $\begin{array}{c}3-8 / 15-8 \\
35-10\end{array}$ & \\
\hline & & $\mathbf{N}$ & 467 & 796 & 545 & \\
\hline & & $\% \mathrm{Oe}+\mathbf{L}$ & 15,4 & 24,7 & 33,9 & 25,1 \\
\hline
\end{tabular}

1. Température maxima absolue - Température minima absolue.

2. Nombre total de cellules construites.

3. Pourcentage des ceufs et des larves morts.

1. Absolute Höchsttemperatur - absolute Tiefsttemperatur.

2. Gesamtzahl der gebauten Zellen.

3. \% abgestorbener Eier und Larven.

La deuxième et la troisième génération d'adultes est plus riche en femelles que la première génération issue des cellules en diapause. La proportion des femelles dans les populations en diapause varie de 12 à $28 \%$, suivant que le lot provient d'un élevage français ou d'un élevage canadien, tandis que la proportion des femelles des deuxième et troisième génération varie de 27 à $82 \%$ en se maintenant généralement aux environs de $30-35 \%$ (tableau 15).

L'analyse détaillée des résultats conduit aux conclusions suivantes :

$1^{0}$ Si l'on considère les nichoirs qui sont comparables, c'est-à-dire ceux qui renferment la progéniture de la première génération issue de parents 
TABL. 15. - Proportion des femelles chez les adultes émergeant avant l'hibernation

Тав. 15. -- Anteil der Weibchen bei den nach Uberwinterung schlüpfenden Adulten

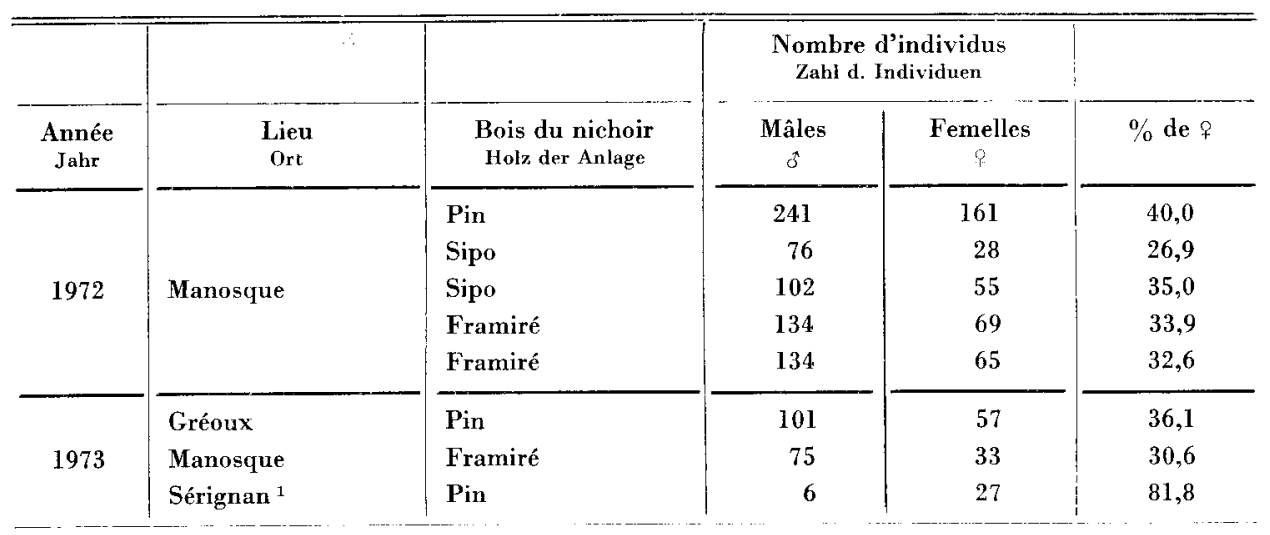

1. Seul nichoir où nidifient les femelles de $2^{e}$ génération.

1. Einzige Anlage, in der die Weibchen der 2. Génération Nester bauen.

canadiens (Manosque et Sérignan, 1972) on obtient dans le bois de Pin les taux d'émergence et les rapports

$$
G^{2} / P N=\frac{\text { Nombre d'insectes émergeant }}{\text { Nombre d'insectes en diapause }}
$$

les plus élevés (tableau 16 et fig. 2). Les résultats des tests de $\chi^{2}$ appliqués aux émergences prouvent que les différences entre bois sont très fortement significatives $\left(\chi^{2}=284\right.$ et 291 pour les nichoirs de Sérignan et de Manosque respectivement). En 1973 à Gréoux et Sérignan on trouve, à nouveau, soit un pourcentage très élevé d'émergences, soit un rapport $\mathrm{G}^{2} / \mathrm{PN}$ élevé dans les nichoirs de Pin (tableau 16).

$2^{\circ}$ La localité de Manosque en 1972 est plus favorable aux émergences que Sérignan bien que les températures moyennes soient toujours plus fortes à Sérignan. Le $\chi^{2}$ testant de façon globale la différence des proportions des individus émergeant dans les deux localités est égal à 12,4. La différence est significative à $99 \%$ de probabilité.

$3^{\circ}$ A Sérignan (1973) le taux d'émergence en troisième génération est très faible $(6 \%)$ alors que le taux d'émergence en deuxième génération est très élevé $(63 \%)$.

$4^{\circ}$ A Manosque (1973) le taux d'émergence en deuxième génération et surtout le rapport $G_{2} / P N$ sont inférieurs aux taux et rapports enregistrés dans les nichoirs de même bois à Manosque en 1972. L'élimination systématique des individus émergeant en seconde génération semble done influer sur le type de développement de la progéniture lors de la génération suivante. 
ÉleVage De Megachile PaCifica




TABL. 17. - Émergence et diapause de la progéniture dans les nids operculés en fonction de la date de ponte et des températures moyennes

TAB. 17. - Schlüpfen und Diapause der Nachkommen in verdeckelten Nestern abhängig vom Zeitpunkt der Eiablage und der Durchschnittstemperatur



I. Température moyenne journalière en degrés $C$.

2. Nombre total de cellules construites.

3. Pourcentage de cellules donnant un adulte émergeant en $2^{\mathrm{e}}$ génération.

4. Rapport Nombre de cellules émergeant

$\overline{\text { Nombre de prénymphes en diapause }}$

1. Tagesdurchschnitt der Temperaturen in Celsiusgraden.

2. Gesamtzahl der gebauten Nester.

3. \% der Zellen, aus denen eine Adulte in d. 2. Generation schlüpfte.

4. Verhältnis der Zahl Zellen mit schlüpfenden Tieren zur Zahl d. Pronymphen mit Diapause. 
$5^{\circ}$ Le tableau 17 (bâti sur le même modèle que le tableau 14) et la figure 4 mettent en évidence l'in fluence de la date de ponte sur l'évolution de la pro. géniture. Dans tous les lieux et pour 4 élevages différents, la période $I$ est très favorable aux émergences et défavorable à l'induction de la diapause tandis que les périodes II et III le sont beaucoup moins. Ceci explique d'ailleurs le fort pourcentage de femelles observé dans les vols de deuxième génération (cf. : tableau 15). En effet, la majorité des femelles est issue de la ponte qui a lieu en début de nidification, alors que la majorité des mâles est issue de la ponte de fin de nidification. L'analyse statistique porte sur chacun des nichoirs; tous les $\chi^{2}$ sont compris entre 11 et 40 , vérifiant ainsi avec la probabilité de $99 \%$ l'hétérogénéité des taux d'émergence au cours de la nidification. Il n'y a aucune relation entre la température moyenne de chaque période et le taux d'émergence correspondant.

$6^{0}$ Dès le début de la période de stockage, le nichoir en Pin de Gréoux 1973 est partagé en deux parties égales : l'une reste à la température du laboratoire, $20-23^{\circ} \mathrm{C}$, l'autre à $19^{\circ} \mathrm{C}$ en chambre climatisée. Au bout d'un mois les adultes ayant émergé dans les deux cas sont comptés : il n'y a aucune différence entre les taux d'émergences correspondant aux deux traitements.

Le bois du nichoir influence de façon incontestable et importante la proportion des individus qui émergent en deuxième génération et la proportion de ceux qui restent en diapause. Le Pin favorise la sortie en deuxième génération. Quelque soit le bois, c'est la ponte de début de nidification qui fournit des individus sans diapause dans la plus forte proportion. Lorsque cette deuxième génération a la possibilité de nidifier, la progéniture qui en est issue donne très peu d'émergences en troisième génération. Enfin il semble que si l'on élimine dans une population “ parent » les individus de la deuxième génération partielle, la progéniture « fille » a tendance à donner une deuxième génération annuelle moins abondante. Cette sélection accroît le rapport

$$
\frac{\text { prénymphes en diapause }}{\text { adultes de } 2^{e} \text { génération }}
$$

\section{DISCUSSION}

Les résultats obtenus au cours de deux ans d'expériences dans le Sud-Est de la France doivent être confrontés avec les données des auteurs américains et européens qui concernent les problèmes de la réactivation, de l'activité des femelles, des possibilités de multiplication, de la mortalité et de la diapause.

\section{I. - La réactivation}

Les températures de réactivation possibles sont d'après STEPhen et Osgoon (1965) comprises entre $19^{\circ} \mathrm{C}$ et $38^{\circ} \mathrm{C}$. L'optimum de $32^{\circ} \mathrm{C}$ permet 
d'obtenir les adultes dans les meilleurs délais, de façon groupée. Cette température réduit au minimum la mortalité et le phénomène de persistance de la diapause. Telford, Johansen et Eves (1972) recommandent avec HobBs (1967 et 1973) la température de $29{ }^{\circ} \mathrm{C}$ dans une ambiance à $60 \%$ d'H.R. Le temps de la réactivation est aussi discuté : HoBBs (1973) recommande d'attendre pour libérer les abeilles dans le champ, qu'il y ait $65 \%$ des mâles et $12 \%$ des femelles émergées dans les (éclosoirs », tandis que Cooke et Dalgleish (non publié) pensent que si les températures nocturnes sont basses il est préférable d'attendre l'émergence de tous les adultes et de pratiquer un nourrissage au sirop de sucre à $20 \%$. Dans nos élevages il semble plutôt intéressant de réactiver les cellules en étuve jusqu'à l'apparition des premiers mâles, puis de fournir dans le champ une humidification correcte des cocons par une technique qui reste à mettre au point.

\section{II. - L'activité des femelles et les possibilités de multiplication}

L'avis des auteurs sur la température seuil pour le vol des femelles est assez partagé : pour ERICKson et WiLson (1972) les femelles ne sont actives que si la température de l'air est supérieure à 24 ${ }^{\circ} \mathrm{C}$. Pour Lee (1967) le seuil varie de $25,5^{\circ}$ à $26,5^{\circ}$ selon la nébulosité du ciel mais il s'abaisse à $24{ }^{\circ} \mathrm{C}$ en septembre chez la deuxième génération. HobBs (1965) estime qu'il y a un début d'activité à $21^{\circ} \mathrm{C}$. L'étude de Szabo et Sмiтh (1972) met en évidence l'interraction lumière-température; ces auteurs trouvent que la gamme des températures seuil s'étend de $17^{\circ} \mathrm{C}$ à $25^{\circ} \mathrm{C}$ selon que la lumière réfléchie est forte (6452 lux) ou faible (1075 lux). Ces mêmes auteurs (1970) estiment que $M$. pacifica a une activité maximum si la température atteint $30^{\circ} \mathrm{C}$. L'espèce est donc exigeante en température et en lumière. Certains espèrent sélectionner une lignée peu exigeante (НовBs, 1973).

La capacité de ponte des femelles est liée à différents facteurs, mais principalement au climat. BoHART, (1962) estime que la fécondité moyenne de l'espèce est 35 œufs par femelle ce qui est élevé pour une abeille solitaire. Les estimations des autres auteurs sont proches de celle-ci (STEPHEN, 1961; Klostermeyer, 1964; Erickson et Wilson, 1972). Lee (1967) observe des variations individuelles de 24 à 72 œufs par femelle. Telford, Johansen et Eves (1972), indiquent une fécondité assez faible : 12 à 15 œufs, qui est proche de celle qui est observée en France (10 à 17 œufs).

Le taux de multiplication du nombre de cellules d'une année à l'autre est la résultante d'un grand nombre de facteurs, il indique la bonne ou mauvaise adaptation de l'espèce à un environnement donné. Les auteurs américains parlent d'accroissements de population impressionnants : dans le Colorado Erickson et Wilson (1972), signalent des rapports d'accroissement annuel de 10. Вонакт (1962), pense que l'on est optimiste si l'on espère un rapport 
de 5. Cet auteur estime que les rapports d'accroissement annuel se situent d'ordinaire entre 2 et 5. En Espagne, Asensio et Rodriguez, (1972) relatent les effets d'un été frais sur le taux de la multiplication. Ce taux est de 0,17 si l'on tient compte des pertes diverses subies par la progéniture (plus de $50 \%$ ). En Hongrie, Manninger (communication personnelle) obtient sur 3 ans des taux moyens de 1,3 à 3 . En France les taux d'accroissement réel sont faibles : 0,2 en année fraîche, 0,6 en année chaude. Cependant les taux d'accroissement apparent respectivement 1,25 et 1,36 sont encourageants. Il s'agit de résultats obtenus seulement par l'élevage de la première génération et l'on peut espérer atteindre un taux d'accroissement réel égal à 2 en laissant nidifier la deuxième génération et en prenant des précautions techniques pour éviter les pertes d'adultes et de larves.

\section{III. - Les ennemis naturels}

L'intérêt des auteurs américains pour les ennemis naturels de $\boldsymbol{M}$. pacifica s'accroît au fil des ans probablement du fait de l'extension des élevages en plein champ et de la manipulation d'un nombre croissant de cellules qui favorisent les attaques et la multiplication des parasites et des prédateurs. Ainsi Stephen et Torchio affirment en 1961 que dans l'Orégon et l'Idaho aucun ennemi indigène n'attaque $M$. pacifica pendant la période de nidification. Ces auteurs pensent que le mégachile a été introduit aux U.S.A. sans ses ennemis naturels, mais en fait, deux chalcidiens : Monodontomerus obscurus et Pteromalus venustus importés d'Europe, sont à présent bien implantés dans les élevages américains de $M$. pacifica. Pour avoir un inventaire complet des parasites et prédateurs de $M$. pacifica on consultera les publications de LEE (1967), Walstrom et Jones (1968), Johansen et al (1969), Johansen et Eves (1966 et 1971), Waters (1971), Telford, Johansen et Eves (1972). Bohart (1972) fait une synthèse des diverses observations effectuées sur le parasitisme en Amérique et cite les espèces suivantes : les coléoptères : Tribolium audax, Trichodes ornatus, le dermaptère : Forficula auricularia, les lépidoptères : Vitula edmandsae et Plodia interpunctella, les hyménoptères : Monodontomerus obscurus, Tetrastichus megachilidis, Melittobia chalybii, Pteromalus venustus, Dibrachys maculipennis, Sapyga pumila, Coelioxys sp., Leucospis affinis. En Europe deux publications seulement communiquent l'inventaire des ennemis de M. pacifica : Asensio et Rodriguez (1972) et Manninger (1972). Pour, les premiers, en Espagne les ennemis sont par ordre d'importance : Miltogramma oestraceum Fll. (15\% d'attaque), Monodontomerus sp. Coelioxys rufocaudata Sm., Trichodes octopunctatus ( $3 \%$ d'attaque) et Zonitis immaculata 01. Pour l'auteur hongrois les principaux ennemis de l'abeille sont Coelioxys rufocaudata et Melittobia acasta; MANNINGER estime que Trichodes apiarius et Anthrenus sp. sont des destructeurs de nids relativement dangereux; il cite 
comme parasites secondaires : Leucospis dorsigera, Sapyga 5 punctata, 2 espèces de Chrysis et Ptinus fur. Le catalogue des ennemis de $M$. pacifica que j'ai établi après deux ans d'observations en France n'est donc pas surprenant. Seules deux nouveautés sont à signaler : le parasitisme des femelles par le diptère conopide Physocephala pusilla et la présence dans les nids d'un cleptoparasite du genre Stelis. Par ailleurs il est bon de souligner l'importance des attaques de Trichodes car ce genre n'est pas une seule fois considéré par les auteurs comme l'ennemi principal.

La biologie des ennemis naturels les plus dangereux pour $M$. pacifica est étudiée dans les publications américaines : le travail de PECK (1969) porte sur 4 hyménoptères : Monodontomerus obscurus, Dibrachys maculipennis, Pteromalus venustus et Melittobia chalybii, celui d'Eves (1970) sur M.obscurus, celui de HobBs et Krunic (1971) sur M. chalybii, P. venustus et $M$. obscurus. Parallèlement des techniques de piégeage sont mises au point et HoвBs (1968) préconise l'utilisation de lampes à U.V. pour capturer dès leur émergence : $M$. obscurus, P. venustus et les Coelioxys. Tonchio (1970 et 1973) entreprend l'étude du développement et du comportement de Sapyga pumila et expérimente trois types de pièges : le piège à $U . V$., le site artificiel d'accouplement recouvert de glue et le gite artificiel de repos nocturne enduit d'insecticide. Les coléoptères nuisibles peuvent être également éliminés : WATERs (1968) décrit un dispositif-piège empêchant les adultes de sortir des ( éclosoirs » en même temps que les abeilles. BoHart (1972) recommande le tamisage des cellules pour éliminer les Trichodes et les Nemognatha. JoHANSEN et Eves (1971) préconisent l'emploi d'un piège à appât de pollen construit avec des plaques de carton ondulé superposées, pour capturer les coléoptères du genre Trogoderma pendant la période de vol des abeilles. Cette méthode rejoint la tentative de piégeage de Trichodes apiarius que j'ai eu l'occasion de faire en 1973 dans un abri à mégachiles. Il est évident que ce système préventif n'est utilisable que dans la mesure où le pollen et les cavités offertes sont attractives pour les femelles du prédateur et les incitent à pondre. Bien qu'il n'y ait aucune observation directe il semble bien que les Trichodes recherchent les nids approvisionnés par les Apoïdes et pondent à l'intérieur. Sur ce sujet on peut lire les remarques de Cros (1908), Marechal (1933) et Gerber (1969) concernant respectivement Trichodes umbellatarum, $T$. alvearius et $T$. ornatus. La recherche du piège à Trichodes le plus efficace reste à faire après l'étude analytique du comportement de ponte de Trichodes apiarius.

\section{IV. - La mortalité des aufs et des larves}

Pour des raisons parfois difficiles à connaître un grand nombre d'œufs et de larves de mégachiles meurt. Ces pertes dues à des causes indéterminées sont observées chez d'autres Megachilidae, par exemple chez les Osmies 
(Maeta et Kitamura - 1968, Danks - 1971, Tasei - 1972 et 1973, Raw - 1972). Chez $M$. pacifica les élevages peuvent subir ainsi plus de $50 \%$ de pertes aux U.S.A. Plusieurs auteurs tentent d'expliquer ce phénomène et proposent parfois des solutions pour réduire la mortalité.

L'action prolongée de températures élevées (supérieures à $39^{\circ} \mathrm{C}$ ) provoque la mort des cufs et des larves. Depuis longtemps on recommande dans les zones chaudes de prendre certaines précautions : disposer un toit ré fléchissant sur l'abri, permettre la circulation de l'air autour des nichoirs à l'intérieur de l'abri.

Bohart (1972) relate que la mortalité est toujours inférieure dans les zones où la luzerne n'est pas la seule plante dont disposent les abeilles. Pour certains auteurs la différence pourrait s'expliquer par la valeur nutritive du pollen de luzerne plus faible que celle du pollen de mélilot par exemple. BoHarT indique que certaines recherches (résultats non publiés) infirment cette hypothèse. On doit la découverte de la véritable cause à Thorp et Briggs (1971) qui démontrent que l'élévation du taux de mortalité dans les zones où abonde la luzerne est due à la richesse en saponines (glycosides solubles dans l'eau) des feuilles de luzerne découpées par les femelles pour construire leurs cellules. Ces auteurs constatent que l'action toxique des saponines est renforcée par les températures élevées. Ils conseillent donc la sélection de variétés de luzerne pauvres en saponines, ou bien l'élevage pour la multiplication des mégachiles sur des papilionacées dont le feuillage est pauvre en saponines : Lotus, Melilotus, Trifolium.

Une cause importante de mortalité chez les stades immatures est signalée par Waller (1969). Les résidus d'insecticides utilisés avant la floraison, tels que D.D.T., Carbaryl, Azinphos sont très dangereux pour la progéniture car ils sont véhiculés aux nids sur les morceaux de feuille qu'utilisent les femelles. Telford, Johansen et Eves (1972) signalent les mêmes inconvénients lors de l'emploi pendant la floraison des produits : Endosulfan et Toxaphène pourtant considérés comme peu toxiques pour l'Abeille domestique. Ils recommandent plutôt l'utilisation de produits tels que : Phosalone, Trichlorfon, Oxydemeton-methyl.

L'existence de maladies chez $M$. pacifica attire peu l'attention des spécialistes. On remarque seulement trois observations récentes : Moore, Henry et Sмiтh (1972) constatant une corrélation entre la mortalité larvaire et la présence d'inclusions protéiques dans les corps gras, suspectent une maladie virale. Quant à Asensio et Rodriguez (1972) et Holm et Skou (1972) ils isolent sur un grand nombre de larves mortes des champignons du genre Ascosphaera.

Les effets létaux de gènes à l'état homozygote sont mis en cause par Gerber (1969). D'après cet auteur l' “ inbreeding » prolongé de certains 
élevages favoriserait l'homozygotie et la mortalité. Par ailleurs l'isolement d'une population risque d'accroître le pourcentage de mâles diploïdes qui ont de faibles chances de survie. Ce phénomène est mentionné par RutTner (1968) chez l'hyménoptère Habrobracon. GERBER recommande d'introduire périodiquement des mâles d'autres régions afin d'accroître le brassage des gènes.

Pour ma part je ne constate pas dans les élevages français de taux aussi élevé de mortalité chez les stades ouf et larve puisqu'il ne dépasse pas $35 \%$ des cellules dans le cas le plus défavorable à la survie. Pour expliquer les différences de mortalité entre localités, entre les nichoirs et entre les périodes de nidification on peut envisager l'action de plusieurs facteurs; respectivement : les basses températures nocturnes $\left(6^{\circ} \mathrm{C}\right)$ provoquant davantage de pertes à Manosque, des émanations nocives propres aux bois exotiques accroissant la mortalité dans les nichoirs en Sipo ou en Framiré, une plus grande sensibilité des mâles aux éléments défavorables à la survie accentuant les pertes en oufs et larves dans la deuxième moitié de la période de nidification. Deux autres causes de mortalité ne peuvent pas être discutées tant que des observations et une expérimentation ne viennent pas démontrer leur effet sur les stades immatures dans les conditions françaises : les saponines et les traitements insecticides effectués avant la floraison. Dans ce but il serait intéressant d'une part d'évaluer le pourcentage de feuilles de luzerne utilisées pour la construction des nids et d'étudier sa fluctuation en fonction de la dimension du champ de luzerne et de son environnement botanique; d'autre part d'expérimenter sur les effets des résidus d'insecticides (Lindane, Parathion, Fenthion) utilisés sur luzerne avant floraison.

\section{V. - La Diapause}

Suivant les auteurs, $M$. pacifica a une, deux ou même trois générations par an selon le lieu et selon l'année. Tous les observateurs reconnaissenć que les générations successives sont partielles, les émergences affectant seùement un certain pourcentage des ccllules de la génération précédente. Lors du vol de deuxième génération le pourcentage d'émergence est $20 \%$ à Washington selon Telford, Johansen et Eves (1972), $0 \%$ dans l'Oregon selon Stephen et Torchio (1961), 4,6\% au Canada selon Krunic (1972), $26 \%$ en Espagne selon Asensio et Rodriguez (1972). Lee (1967) observe qu'une même année le taux d'émergence varie de 4 à $24 \%$ suivant la localité. Les explications et les hypothèses que fait naître la question de la diapause prénymphale chez $M$. pacifica ne manquent pas.

Une idée couramment admise est que la diapause de cette espèce dépend de la température à laquelle est soumise la progéniture pendant son développement (Stephen et Osgood, 1965 b., Krunic, 1972, Krunic et Hinks 1972). Ainsi Stephen (1961) observe une deuxième génération, dans les nichoirs 
exposés au soleil, et une seule génération dans les nichoirs abrités. D'autres hyménoptères entrent en diapause de cette manière, après avoir subi pendant le développement de l'œuf et de la larve des températures peu élevées, c'est le cas de Spalangia drosophilae (SImmonos, 1948) et de Tritneptis klugii (Schneiderman et Horwitz, 1958).

Un autre facteur inducteur de la diapause est l'âge de la femelle : JoHANSEN et Eves (1973) observent que la plus forte proportion d'émergences survient dans les cellules construites au début de la période de nidification, et ceci quelle que soit la date du lâcher des abeilles. Les femelles jeunes ont tendance à pondre des oufs donnant des larves sans diapause. Quand elles vieillissent les œufs pondus donnent de plus en plus de larves à diapause quelles que soient les conditions du milieu. Chez les hyménoptères Mormoniella vitripennis et Spalangia drosophilae Saunders (1962) et Simmonds (1948) démontrent également que la diapause de la progéniture est in fluencée par l'âge de la mère.

Johansen et Eves pensent que la photopériode n'a pas d'action sur les femelles et n'entre pas en jeu de façon significative dans le déterminisme de la diapause. En effet les courbes d'émergence de deux élevages faits à un mois d'intervalle sont pratiquement identiques.

L'étude de l'in fluence du génotype sur la diapause est en cours au Canada. Новвs (1965) estime que le fait d'éliminer les adultes émergeant en deuxième génération doit permettre à l'éleveur de mégachiles d'obtenir peu à peu une lignée monovoltine. KRunic (1972) trouve que très peu de femelles donnent des adultes en deuxième génération et suppose qu'il existe un facteur héréditaire de diapause. Il faut ici rappeler que le seul insecte dont on a étudié la génétique de la diapause est Bombyx mori le ver à soie (TANAKa - 1953). L'étude consiste à démontrer comment le génotype des individus modifie la réponse du c mécanisme récepteur ) des facteurs du milieu, tel que la température (LEEs - 1956). Chez diverses espèces on est arrivé par la sélection, à isoler les lignées mono ou multivoltines, dans des populations renfermant les deux types d'insectes. LE BERre (1953) sélectionne 2 lignées de Locusta migratoria (une monovoléine, l'autre bivoltine) dans une population naturelle. DANYLEvskir (1965) signale qu'Arbuthnot isole en 1944 une race monovoltine du lépidoptère : Pyrausta nubilalis et Prebree en 1941 une race polyvoline de Gilpinia polytoma.

Dans les élevages français de $M$. pacifica de 1972 et 1973 il n’est guère possible de distinguer une action de la température sur la diapause. Par contre l'in fluence de la sélection pour une lignée monovoltine somble se manifester dès la première année. La poursuite de l'expérimentation pourrait confirmer l'hypothèse du contrôle génétique de la diapause. Le facteur âge des femelles s'exprime de façon très nette dans tous les élevages, comme dans les données provenant d'Amérique. L'in fluence du bois du nichoir est aussi évidente mais on ne trouve pas dans la littérature de remarque semblable concernant $M$. paci- 
fica ou quelqu'autre apoïde. Cette observation est à rapprocher de celles de Carayon et Thouvenin (1966) et de Slama (1969). Ce dernier fait état de la découverte de substances analogues aux hormones de développement des insectes (hormone juvénile ou ecdysone) dans les végétaux. D’après Carayon et Thouvenin ces substances agiraient par contact aussi bien que par émanation. Il est donc possible que les nichoirs de Pin soient le siège de fortes émanations de produits analogues à l'ecdysone, ce qui expliquerait la métamorphose d'un grand nombre de prénymphes en nymphes et la sortie d'une abondante deuxième génération dans ces nichoirs. On doit noter aussi l'évolution bien différente des deux générations : la première donnant une progéniture à taux élevé d'émergences, la deuxième donnant une progéniture à taux très faible. Il s'agit soit d'un effet du changement de l'environnement (température par exemple) soit d'une réponse différente de la deuxième génération à un même environnement. La deuxième génération différant de la première par la nervation (KRUNIC, 1972) on peut imaginer qu'elle en diffère aussi par la physiologie.

Bien que le terme de diapause soit ambigu, on voit après cette discussion que l'arrêt de développement de $M$. pacifica présente nettement plusieurs caractères d'une diapause “ sensu lato » : le stade d'arrêt de développement est toujours le même; des processus hormonaux sont en jeu car d'une part l'induction de cet arrêt est in fluencée par l'âge de la mère et d'autre part les insectes en repos ont besoin d'éliminer leur état de vie ralentie au cours d'une période de froid (température optimum comprise entre 5 et $15^{\circ} \mathrm{C}$ ). Par ailleurs si la température est trop basse pendant l'hibernation, ou bien s'il y a alternance des températures pendant la réactivation l'état de vie ralentie est renforcé. Enfin si la période de froid n'a pas eu lieu $50 \%$ seulement des insectes en repos émergent dans les délais anormalement longs et d'une façon anormalement échelonnée dans le temps (voir à ce sujet KRUnic et Hinks, 1972 et Stephen et Osgood, 1965 b.). Cependant aucun auteur ne met en évidence l'in fluence de la photopériode et il est plutôt question de l' " accumulation 》 d'un facteur défavorable à la poursuite du développement : les basses températures. Il ne s'agirait donc pas chez $M$. pacifica d'une diapause (c sensu stricto ) ou “ eudiapause » suivant la terminologie de MüLLER (1970) mais d'un arrêt de développement d'un type proche de c l'oligopause-diapause » de Müller bien que l'induction soit due aux basses températures et non à la photopériode. Beaucoup de points restent à éclaircir concernant :

a) le mode d'action de la température (seuil, durée d'action, stade réceptif),

b) le caractère héréditaire de la sensibilité aux basses températures,

c) l'hypothèse de la présence de substance analogue à l'ecdysone dans le bois de Pin, et de son action lorsque les cellules sont construites dans un nichoir de Pin. 
La diapause de $M$. pacifica est non seulement une question d'intérêt fondamental mais aussi un problème pratique d'élevage puisque l'émergence d'une deuxième génération peut être suivant l'environnement et la technique employée, une cause de pertes ( $d$ 'autant plus sévère que les femelles sont nombreuses) ou au contraire un facteur d'accroissement de la population. Dans l'exemple relaté au chapitre des résultats il semble que l'élevage d'une deuxième génération soit plus favorable à la multiplication de l'espèce, que celui d'une lignée monovoltine. Si cette tendance se confirmait il serait plus rentable de chercher à obtenir une lignée strictement bivoltine.

\section{CONCLUSION}

Les élevages en France de $M$. pacifica importé du Canada permettent d'analyser les effets de quelques facteurs écologiques sur l'activité de nidification, la mortalité et la diapause de l'écotype américain. Technique d'élevage et facteurs de l'environnement sont souvent des causes de régression des populations.

1. Une réactivation trop longue en étuve peut causer des pertes importantes chez les adultes. Si elle est trop courte, il y a des risques accrus de mortalité par dessication chez les prénymphes et les nymphes apportées sur le lieu du lâcher.

2. Les facteurs climatiques : vent violent, température inférieure à 24-25 ${ }^{\circ} \mathrm{C}$ sont causes respectivement de pertes chez les adultes et de ralentissement de l'activité des femelles. La ponte moyenne est 10 œufs par femelle en année fraîche et 17 en année chaude.

3. Les ennemis naturels indigènes sont de sérieux facteurs de pertes. Le ravageur le plus constant et le plus actif est Trichodes apiarius (jusqu'à $34,7 \%$ des cellules construites) Miltogramma murinum et Melittobia acasta sont des espèces secondaires pouvant prendre de l'extension.

4. Les bois exotiques Sipo et Framiré utilisés dans la construction de nichoirs ont une in fluence néfaste sur la survie des œufs et des larves d'abeilles. Les effets d'autres facteurs de mortalité des stades immatures : taux élevé de saponines dans les feuilles de luzerne, températures nocturnes basses, maladies, résidus d'insecticides, ne sont pas étudiés.

5. Lorsque l'on fait nidifier seulement la première génération, une partie parfois très importante de la progéniture émerge en deuxième génération pendant la période de stockage (jusqu'à $64,5 \%$ des cellules construites) : Les taux d'émergences les plus élevés sont observés dans les nichoirs en bois de Pin. On suspecte ce bois de renfermer une substance analogue à l'ecdysone. Les bois exotiques (Sipo et Framiré) en seraient dépourvus ou en posséderaient relativement peu. Quelles que soient les conditions du milieu, les émergences 
affectent surtout la descendance issue des premiers œufs pondus. L'élimination des insectes sans diapause paraît accroître le nombre d'insectes en diapause l'année suivante. Par contre si on laisse les individus sans diapause nidifier en fin de saison dans les nichoirs, le taux d'accroissement de la population n'est pas négligeable $(1,3)$ malgré la raréfaction des plantes fleuries. La descendance des femelles de deuxième génération donne un très faible pourcentage d'individus de troisième génération.

6. La multiplication des cellules $d$ 'abeilles d'une année sur l'autre est relativement peu élevée. En utilisant seulement la première génération, les meilleurs taux d'accroissement brut sont 1,2 et 1,4 . Si l'on déduit les pertes, ils sont inférieurs à $1:(0,2$ et 0,6$)$.

La recherche des meilleurs moyens permettant d'accroître les populations. de $M$. pacifica doit conduire à :

1. améliorer les techniques de réactivation des cellules et de construction des abris,

2. étudier la biologie des ennemis naturels les plus dangereux,

3. analyser les effets des principaux facteurs de mortalité des adultes et des stades immatures (insecticides, saponines, maladies),

4. expérimenter sur les facteurs inducteurs de la diapause (température, génotype, substances analogues des hormones de développement),

5. observer l'utilisation par les femelles de mégachiles de l'environnement botanique (feuilles et pollens) au cours de la saison.

On peut espérer contrebalancer l'effet de certains facteurs en prenant certaines précautions : lors de la réactivation soit en nourrissant artificiellement les adultes, soit en humidifiant les cellules; lors de la nidification en utilisant uniquement le bois de Pin favorisant la survie des larves, en protégeant l'abri avec un brise-vent et en piégeant les Trichodes. A long terme on peut envisager la création d'une lignée de mégachiles monovoltins ou bivoltins ou encore d'une lignée active à une température d'environ $20^{\circ} \mathrm{C}$.

Cependant dans les conditions actuelles le facteur température, limitant l'expression du potentiel de ponte des femelles ne laisse guère l'espoir de dépasser un rapport d'accroissement annuel de la population annuel égal à 2, et ceci dans les meilleures conditions de sauvegarde. 


\section{REMERCIEMENTS}

Je remercie la Fédération Nationale des Agriculteurs Multiplicateurs de Semences pour sa collaboration technique et financière.

M. GUY (I.N.R.A., Lusignan) pour ses conseils lors de l'analyse statistique de certains résultats.

M. Desmier de Chenon, d'Aguilar (I.N.R.A., Versailles) et Cantot (I.N.R.A., Lusignan) pour la détermination respectivement, d'hyménoptères, de diptères et de coléoptères.

\section{ZUSAMMENFASSUNG}

Die Blattschneiderbiene Megachile pacifica Panz. (M. rotundata F.) (Foto : Tafel 1 u. 2) ist eine in Frankreich und anderen europäischen Ländern vorkommende paläarktische Art, die etwa um 1930 zufällig aus U.S.A. eingeführt wurde. Dieser Luzernebestäuber ist seit 1960 in weitem Umfang Züchtungsobjekt, besonders in den warmen und trockenen Gebieten Amerikas. 1972 und 1973 wurden Versuche mit aus Kanada eingeführten $M$. pacifica durchgeführt, um die Möglichkeiten der Verbreitung des amerikanischen Ökotypus im Südosten Frankreichs zu ergründen.

\section{Material und Methode}

In der Gegend von Manosque, Sérignan und Gréoux wurden vier Felder mit Samen!luzerne ausgewählt. Die klimatischen Bedingungen sind aus Tabelle 1 ersichtlich. Die Insekten kommen in ihren Kokons als überwinternde Pronymphen in Frankreich an. Nach Vernichtung etwaiger Parasiten werden die Bienen reaktiviert, so dass die Adulten gegen Ende Juni/Anfang Juli schlüpfen. Die Nistanlage entspricht dem kanadischen Muster. Es handelt sich dabei um eine Schutzvorrichtung mit zerlegbaren Nistanlagen aus Kiefern-, Sipo- oder Framiréholz ${ }^{1}$, die $8 \mathrm{~cm}$ lang und mit Löchern von $6 \mathrm{~mm}$ Durchmesser versehen sind (Foto : Tafel 3). Tabelle 2 enthält genaue Angaben über die verschiedenen, im Verlauf von zwei Jahren durchgeführten Versuchsserien.

\section{Ergebnisse}

I. - Bei allen Aufzuchten ist die Zuwachsrate der Populationen ziemlich gering; in den meisten Fällen beträgt sie das 1,4 fache, d.h. das 0,6 fache bei Berücksichtigung der Verluste Tab. 7 u. 8).

II. - Eine zu lange dauernde Reaktivierung im Brutschrank kann bedeutende Verluste bei den Adulten verursachen. Ist dagegen der Aufenthalt im Brutschrank zu kurz, kann die Reaktivierung unter natürlichen Bedingungen im Freien den Tod der Pronymphe und der Nymphe durch Austrocknen nach sich ziehen (Tab. 3).

III. - Der Wind und die verhältnismässig niedrigen Temperaturen von $24-25{ }^{\circ} \mathrm{C}$ sind die Ursache von Sterblichkeit, beziehungsweise Verzögerung der Aktivität der Weibchen (Abb. 1).

IV. - Die einheimischen, natürlichen Feinde sind wichtige Verlustfaktoren (10-50\% der errichteten Zellen) (Abb. 3 u. 3a u. Foto : Tafel 4 u. 5). Trichodes apiarius ist der bei weitem beständigste und schädlichste Feind (bis zu $35 \%$ der Zellen). Miltogramma murinum und Melittobia acasta folgen in zweiter Linie (Tab. 10).

1. Fu snote : Pinus, Entandrophragma und Terminalia. Die beiden letzten sind afrikanische Hölzer. 
V. - Die Sterblichkeit der Frühstadien aus unbekannter Ursache schwankt zwischen $3 \%$ und $35 \%$. Die den Weibchen zum Nestbau gebotenen exotischen Hölzer (Sipo und Framiré) scheinen von schädlichem Ein ßuss auf das Überleben der Eier und der Larven, während die Sterblichkeit im Kiefernholz geringer ist (Abb. 3 u. 3a, sowie Tab. 13).

VI. - Wenn man nur die 1. Generation bauen lässt, entwickelt sich ein manchmal beträchtlicher Teil der Nachkommen zu Adulten, die eine 2. Generation bilden (11 \% bis $64 \%$ der gebauten Zellen). Die Nestanlagen im Kiefernholz zeigen in der 2. Generation eine erhöhte Schlüpfrate. Man könnte vermuten, dass dieses Holz eine Substanz, ähnlich dem Ekdyson, enthält, die die afrikanischen Hölzer nicht oder nur in geringerem Masse besitzen und die die. Larven daran hindert, eine Diapause einzulegen (Tab. 16). Das Ausscheiden der Insekten ohne Diapause scheint im darauffolgenden Jahr die Zahl derjenigen, die eine Diapause durchmachen, zu vergrössern.

VII. - Sterblichkeit und Diapause erfassen die Nachkommenschaft in verschiedener Weise, je nachdem ob die Zellen über Eiern gebaut werden, die zu Beginn oder gegen Ende des Nestbaues gelegt wurden (Abb. 4 u. 4a; Tab. 14 u. 17). Die Sterblichkeitsquote sowie der Anteil der Larven mit Diapause wachsen gegen Ende der Eiablage an.

VIII. - Wenn man die Individuen, die keine Diapause eingelegt haben, in der 2. Generation bauen lässt, ist die Zuwachsrate von Generation zu Generation während des Sommers. gleich 1,3, und die Nachkommenschaft der 2. Generation bringt nur einen geringen Prozentsatz an Individuen ohne Diapause (6\%, Tab. 16).

\section{Schlussfolgerungen}

Der Einwirkung gewisser ungünstiger Faktoren auf die Verbreitung von Megachile pacifica könnte vielleicht entgegengearbeitet werden durch Verbesserung der Reaktivierungstechnik und der Konstruktion der Schutzvorrichtungen sowie durch den intensiveren Schutz der Larven vor Trichodes durch Anbringen von Pollenfallen.

Ausserdem müsste man wissen, ob Auswahl und Verwendung einer nur einmal jährlich. Eier legenden Linie eine stärkere Vermehrung erlaubt als die Zucht einer Megachile, die zweimal im Jahr Brut hat. Das Klima scheint allerdings einer optimalen Aktivität der Art nicht günstig zu sein; und man kann nicht erwarten, in jedem Jahr die doppelte Zuwachsrate zu übers chreiten.

\section{REFERENCES BIBLIOGRAPHIQUES}

Asensio de la Sienra E., Roghiguez Ibanez J.-A., 1972. Primer informe sobre adaptacion y multiplicacion de la abeja polinizadora de la alfalfa Megachile pacifica Panz. (Megachile. rotundata auct. nec Fabr.). An. Inst. Nac. Invest. Agrar. Ser. Prot. veg., 2, 237-252.

Aubury R.-G., Rogers H.-H., 1971. The use of Megachile rotundata F. as a pollinator of lucerne in glasshouses. J. Brit. Grassl. Soc., 26, 91-94.

Benedex P., 1968. The flight period of wild bees (Hym. Apoidea) pollinating lucerne and its plant protection aspects. Acta Phytopath. Acad. Sci. Hung., 3, 59-71.

Benedek P., 1969. Examinations on lucerne pollinating wild bees (Hym. Apoidea) in the hungarian great plain. Folia Entomol, hung., 22, 101-115.

Bohart G.-E., 1962. How to manage the leaf-cutting bee (Megachile rotundata F.) for alfalfa pollination. Circ. Utah Agr. Exp. St. N. 144, 7 p.

Bohart G.-E., 1970. Commercial production and management of wild bees. A new entomological industry. Bull. Entomol. Soc. Amer., 16, 8-9.

Bohart G.-E., 1972. Management of wild bees for pollination of crops. Ann. Rev. Entomol., 17, 287-312.

Bohart G.-E. Communication personnelle.

Carayon J., Thouvenin M., 1966. Emploi d'une substance mimétique de l'hormone juvénile pour la lutte contre les Dysdercus, hyménoptère nuisible au cotonnier. C.R. Acad. Agr.,. 52, 340-346. 


\section{Élevage: de megachile pacifica}

Cooke D.-A., Dalgreish J.-M. (non publié). A new incubation system for leafcutter bees. Cros A., 1908. Le Trichodes umbellatarum ol., ses mœurs, son évolution. Bull. Mus. Hist. Nat. Paris, 14, 215-221.

DaLY H.-V., 1952. Records of the palearctic Megachile rotundata in the United States (Hym. Apoidea). Entomol. News, 63, 210-211.

Danks H.-V., 1971. Nest mortality factors in stem-nesting aculeate hymenoptera. $J$. $J$. Anim. Ecol., 40, 79-82.

Danylevskir A.-S., 1965. Photoperiodism and seasonal development of insects. Oliver and Boyd. Edinburgh and London.

Desmier de Chenon R., Communication personnelle.

Erickson E.-H., Wilson W.-T., 1972. Management of the alfalfa leaf-cutter bee in Colorado. Megachile rotundata. Colorado St. Univ. Exper. Stn. Bull. 5525, 17 p.

Ercandsson S., 1960. The occurence of Megachile argentata $\mathrm{F}$. and rotundata $\mathrm{F}$. in Scandinavian countries (Hym.). Entomol. Ts. Stockholm, 81, 25-29.

Eves J.-D., 1970. Biology of Monodontomerus obscurus Westw. a parasite of the alfalfa leafcutting bee, Megachile rotundata (Hym. Torymidae; Megachilidae) Melanderia, 4, 1-18.

Eves J.-D., 1973. Population dynamics of larvae of the alfalfa leafcutting bee Megachile rotundata in eastern Washington (in Diss. Abstr. int., 33, $5890 \mathrm{~B}$ ).

Gerber H.-S. 1969. Factors affecting the sex ratio and nesting behaviour of Megachile rotundata F. (Hym. megachilidae) Ph. D. Washington St. Univ.

Guy P., Lecomte J., Ecalle C., 1969. Utilisation d'insectes pour la pollinisation de la luzerne en enceintes fermées. Ann. Amelior. Plantes, 19, 333-343.

HeINRICHS D.-H., 1967. Seed increase of alfalfa in growth chambers with Megachile rotundata. Canad. J. Plant. Sci., 47, 691-694.

Новвs G.-A., 1965. Importing and managing the alfalfa leaf-cutter bee. Canada Dep. Agric. Pub., 1209, 11 p.

Hoвbs G.-A., 1967. Domestication of alfalfa leaf-cutter bees. Canada Dep. Agr. Pub., 1313 , $19 \mathrm{p}$.

Honss G.-A., 1969. Further information on alfalfa leaf-cutter bee keeping. Canad. Agr. Res. St. Lethbridge, 9 p.

Новвs G.-A., 1973. Alfalfa leaf-cutter bees for pollination of alfalfa in western Canada. Inf. Div. Canada Dep. Agr., 149, 30 p.

Hоввs G.-A., Krunic M.-D., 1971. Comparative behavior of three chalcidoid (Hym.) parasites of the alfalfa leaf-cutter bee, Megachile rotundata, in the laboratory. Monodontomerus obscurus, Pteromalus venustus, Mellitobia chalybii. Canad. Entomol., 103, 674685.

Holm S.-N., Skou J.-P., 1972. Studies on trapping, nesting and rearing of some Megachile species (Hym. Megachilidae) and their parasites in Denmark Entomologica Scandinavia, 3, $169-180$.

HuRd P.-D., 1954. Distributional notes on Eutricharea, a paleartic genus of Megachile, which has become established in the United States. Entomol. News, 65, 93-95.

Hund P.-D., 1967. The identity of Megachile rotundata F. and Megachile argentata F. ( $\mathrm{Hym}$. Apoidea). Entomol. Medd., 35, 3-10.

Johansen C.-A., 1971. How to reduce poisoning of bees from pesticides. Wash. St. Univ. Coop. Ext. Serv. E.M. 3473, 6 p.

Johansen C.-A., Eves J.-D., 1966. Parasites and nest destroyers of the alfalfa leaf-cutting bee. Wash. Agr. Exper. Stn. Circ, 469, 12 p.

Johansen C.-A. et al., 1969. Suggestions for alfalfa leaf-cutter bee management. Coop. Ext. Serv. Wash. St. Univ. Pullman. EM, 2775 Revised, 8 p.

Johansen C.-A., Eves J.-D., 1971. Control of alfalfa leaf-cutter bee enemies. Wash. St. Univ. Agr. Ext. Serv. E.M., 263110 p.

Jonansen C.-A., Eves J.-D., 1973. Effects of chilling, humidity and seasonal conditions on emergence of the alfalfa leaf-cutting bees. Environ. Entomol., 2, 23-26.

Klostermeyer L.-E., Soo C.-F., 1968. Chromosome number in the leaf-cutter bee. Megachile rotundata. Ann. Entomol. Soc. Amer., 61, 782-183.

Krombein K.-V., 1948. An adventive Megachile in Washington. D.C. Entomol. Soc. Wash. Proc., 50, 14.

KruNIC M.-D., 1972. Voltinism in Megachile rotundata (Megachilidae : Hym.) in southern Alberta. Canad. Entomol., 104, 185-188.

Krunic M.-D., Hinks C.-F., 1972. The effect of temperature and of temperature pretreatment on diapause and on the synchronization of adult emergence in Megachile rotundata (Hym. Megachilidae). Canad. entomol., 104, 889-893.

Kukovica I., 1966. A study of the reproductive capacity, foraging behaviour and environmen- 
tal adaptability of the leaf-cutting bee Megachile rotundata in southern Ontario. M. Sci. thesis Univ. Guelph.

Le Berre J.-R., 1953. Contribution à l'étude biologique du Criquet migrateur des Landes. (Locusta migratoria gallica Rem.) Bull. Biol. Fr. Belg., 87, 227-273.

LEComte J. Communication personnelle.

LEE S.-L., 1967. Biology of the leaf-cutter bee Megachile rotundata in New York State. Ph. D. thesis Cornell Univ.

LeEs A.-D., 1956. The physiology and biochemistry of diapause. Ann. Rev. Entomol., 1, 1-16.

Maeta Y., Kitamura T., 1968. Some biological notes on the introduced wild bee, Osmia (Osmia) lignaria Say. (Hym. Megachilidae). Bull Tohoku Nat. Agric. Exper. Stn., 36, 53-70.

Manninger S., 1972. Parasites of alfalfa leaf-cutter bee, Megachile rotundata F. (M. pacifica Danz.) in Hungary. Novenytermeles, 21, 321-328.

ManNinger S. Communication personnelle.

Maréchal P., 1933. Sur quelques habitants des chanmes des toitures Eriades maxillosus $L$. et ses parasites (surtout Trichodes alvearius F.). Bull. Ann. Soc. Entomol. Belg., 227257.

Moczar L., 1959. The activity of the wild bees in hungarian lucerne fields. Acta Agron., 9, $237-289$.

Moore J.-I., Henry J.-E., SMith H.-W., 1972. Inclusions in the fat body of the alfalfa leafcutter bee Megachile rotundata. Ann. Entomol. Soc. Amer., 65, 1268-1289.

Muller H.-J., 1970. Formen der Dormanz bei Insekten. Nova Acta Leopoldina, 191, 7-25.

Osgood C.-E., 1968. Mechanisms of orientation in the leaf-cutterbee, Megachile rotundata. Ph. D. thesis Oregon St. Univ. Corvallis.

Packer J.-S., 1971. The flight and foraging behaviour of the alkali bee (Nomia melanderi). and alfalfa leaf-cutter bee (Megachile rotundata). Ph. D. thesis Utah St. Univ. Logan.

Peck O., 1969. Chalcidoid (Hymenoptera) parasites of the alfalfa leaf-cutter bee, Megachile rotundata in Canada. Canad. Entomol., 101, 418-422.

Raw A., 1972. The biology of the solitary bee Osmia rufa L. (Megachilidae) Trans. R. Entomol. Soc. Lond., 124, 213-229.

Rebmann O., 1967. Beitrag zur Kenntnis der Gattung Megachile Latr. (Hym. Apidae) Was ist " Megachile argentata auct. ") und " Megachile rotundata auct. ) Entomol. Z. Stuttgart. 77, $169-17$.

Rutwner F., 1968. in Traité de biologie de l'Abeille 4, 198-236. Masson, Paris.

Saunders D.-S., 1962. The Effect of the age of female Nasonia vitripennis (Walker) (Hym., Pteromalidae) upon the incidence of larval diapause. J. Ins. Physiol., 8, 309-318.

Schneiderman H.-A., Horwitz J., 1958. The induction and termination of facultative diapause in the chalcid waps Mormoniella vitripennis Walk. and Tritneptis klugii Ratz. J. Exper. Biol., 35, 520-551.

Simmonds F.-J., 1948. The influence of maternal physiology on the incidence of diapause. Philos. Trans. B., 233, 385-414.

Slama K., 1969. Plants as source of materials with insect hormone activity. Entomol. Exper. Appl., 12, 721-728.

Solrnas M., Bin F., 1965. Osservazioni sugli insetti impollinatori delle leguminose foraggere Medicago sativa e Trifolium pratense nelle Pianura Padena. Semeni Elette, 11, 1-32.

Stephen W.-P., 1961. Artificial nesting sites of the propagation of the leaf-cutter bee $M e g a-$ chile (Eutricharea) rotundata for alfalfa pollination. J. Econ. Entomol., 54, 989-993.

Stephen W.-P., 1962. Propagation of the leaf-cutter bee for alfalfa seed production. Oreg. Agr. Exp. Stn. Bull., 586, 16 p.

Stephen W.-P., Tonchio P.-F., 1961. Biological notes on the leaf-cutting bee, Megachile (Eutricharea) rotundata. Pan. Pac. Entomol., 37, 85-93.

Stephen W.-P., Osgood C.-E., 1965 (a). Influence of tunnel size and nesting medium on sex ratios in a leaf-cutter bee Megachile rotundata. J. Econ. Entomol., 58, 965-968.

STEphen W.-P., Osgoon C.-E., 1965 (b). The induction of emergence in the leaf-cutter bee Megachile rotundata an important pollinator of alfalfa. J. Econ. Entomol., 58, 284-286.

Szabo T.-I., Sмiтh M.-V., 1970. The use of Megachile rotundata for the pollination of greenhouse cucumbers. Rep. IX Pollination Conf., 95-105.

Szabo T.-I., Smith M.-V., 1972. The influence of light intensity and temperature on the activity of the alfalfa leaf-cutter bee Megachile rotundata under field conditions. $J$. Apic. Res., 11, 157-165.

Tanaka J., 1953. Genetics of the silkworm, Bombyx mori. Advanc. Genet., 5, 239-317.

TAsEI J.-N., 1972. Observations préliminaires sur la biologie d'Osmia coerulescens L. pollinisateur de la luzerne. Apidologie, 3, 149-165. 
TAsei J.-N., 1973. Observations sur le développement d'Osmia cornuta Latr. et Osmia rufa L. (Hym. Megachilidae). Apidologie, 4, 295-315.

Telford H.-S., Johansen C.-A., Eves J.-D., 1972. Management practices and insecticide poisoning of Nomia melanderi Chll. and Megachile rotundata F., two valuable pollinators of alfalfa grown for seed in Washington State. Meded. Fac. Landbouxet, Rijksuniv. Gent., 37, 776-783.

ThorP R.-W., Briggs D.-L., 1971. Mortality on immature alfalfa leaf-cutter bees in relation to alfalfa saponins. Megachile rotundata. Environ. Entomol., 1, 399-4.01.

Tirgari S., 1963. The biology and management of the alfalfa leaf-cutter bee Megachile rotundata. M. Sci. thesis Utah St. Univ. Logan.

Torchro P.-F., 1970. The biology of Sapyga pumila Cress. And its importance as a parasite of the alfalfa leaf-cutter bee Megachile rotundata F. Arkansas Agric. Ext. Serv. M. P., $127,84-90$.

Tonchio P.-F., 1973. Sapyga pumila Cress. a parasite of Megachile rotundata F. (Hym. Sapygidae) I. Biology and description of immatures stages. II Methods of control. Entomol. Res. Div. Agr. Res. Serv., 30 p.

WALSTrom R.-J., Jones P.-A., 1968. Alfalfa leaf-cutter bee management for alfalfa pollination in South Dakota. S. Dakota. S. Dak St. Univ. Agr. Exp. Stn. Bull., 544.

W ATERS N.-D., 1968. Alfalfa leaf-cutter bee excluder beetle trap. Idaho Univ. Curr. Inf. Ser. $97 ; 4$ p.

WATERS N.-D., 1971. Insect enemies of the alfalfa leaf-cutter bee and their control. Idaho Univ. Curr. Inf. Ser. 163, $4 \mathrm{p}$.

Wilson E.-B., 1968. Leaf-cutting bee storage. Wash. St. Univ. Ext. Serv. E.M. 2909, 5 p. 